\title{
X-ray follow-ups of XSS J12270-4859: a low-mass X-ray binary with gamma-ray Fermi-LAT association ${ }^{\star}$
}

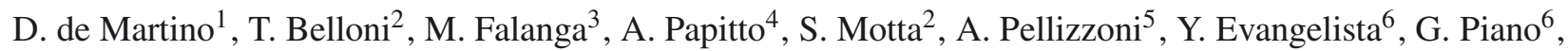
N. Masetti ${ }^{7}$, J.-M. Bonnet-Bidaud $^{8}$, M. Mouchet ${ }^{9}$, K. Mukai $^{10}$, and A. Possenti ${ }^{5}$

1 INAF - Osservatorio Astronomico di Capodimonte, salita Moiariello 16, 80131 Napoli, Italy e-mail: demartino@oacn.inaf.it

2 INAF - Osservatorio Astronomico di Brera, via E Bianchi 46, 23807 Merate (LC), Italy

e-mail: [tomaso.belloni; sara.motta] @brera.inaf.it

3 International Space Science Institute (ISSI), Hallerstrasse 6, 3012 Bern, Switzerland e-mail: mfalanga@issibern.ch

${ }^{4}$ Institut de Ciéncie de l'Espai (IEEC-CSIC) Campus UAB, Fac. de Ciéncies, Torre C5 08193 Barcelona, Spain e-mail: papitto@ice.csic.es

5 INAF - Osservatorio Astronomico di Cagliari, loc. Poggio dei Pini, strada 54, 09012 Capoterra (CA), Italy e-mail: apellizz@oa-cagliari.inaf.it

6 INAF - Istituto di Astrofisica Spaziale e Planetologia Spaziali, via Fosso del Cavaliere 100, 00133 Roma, Italy e-mail: [yuri.evangelista;giovanni.piano]@iasf-roma.inaf.it

7 INAF - Istituto di di Astrofisica Spaziale, via Gobetti 101, 40129 Bologna, Italy e-mail: nicola.masetti@iasfbo.inaf.it

${ }^{8}$ CEA Saclay, DSM/Irfu/Service d'Astrophysique, 91191 Gif-sur-Yvette, France e-mail: bonnetbidaud]@cea.fr

9 Laboratoire APC, Université Denis Diderot, 10 rue Alice Domon et Léonie Duquet, 75005 Paris, France and LUTH, Observatoire de Paris, Section de Meudon, 5 place Jules Janssen, 92195 Meudon, France e-mail: martine.mouchet@obspm.fr

${ }^{10}$ CRESST and X-ray Astrophysics Laboratory, NASA Goddard Space Flight Center, Greenbelt, MD 20771, USA and Department of Physics, University of Maryland, Baltimore County, 1000 Hilltop Circle, Baltimore, MD 21250, USA e-mail: koji.mukai@nasa.gov

Received 17 September 2012 / Accepted 2 December 2012

\section{ABSTRACT}

Context. XSS J1227.0-4859 is a peculiar, hard X-ray source recently positionally associated to the Fermi-LAT source 1FGL J1227.94852/2FGL J1227.7-4853. Multi-wavelength observations have added information on this source, indicating a low-luminosity lowmass X-ray binary (LMXB), but its nature is still unclear.

Aims. To progress in our understanding, we present new X-ray data from a monitoring campaign performed in 2011 with the XMM-Newton, RXTE, and Swift satellites and combine them with new gamma-ray data from the Fermi and AGILE satellites. We complement the study with simultaneous near-UV photometry from XMM-Newton and with previous UV/optical and near-IR data. Methods. We analysed the temporal characteristics in the X-rays, near-UV, and gamma rays and studied the broad-band spectral energy distribution from radio to gamma rays.

Results. The X-ray history of XSS J1227 over 7 yr shows a persistent and rather stable low-luminosity $\left(6 \times 10^{33} d_{1 \mathrm{kpc}}^{2} \mathrm{erg} \mathrm{s}^{-1}\right)$ source, with flares and dips being peculiar and permanent characteristics. The associated Fermi-LAT source 2FGL J1227.7-4853 is also stable over an overlapping period of $4.7 \mathrm{yr}$. Searches for X-ray fast pulsations down to msec give upper limits to pulse fractional amplitudes of $15-25 \%$ that do not rule out a fast spinning pulsar. The combined UV/optical/near-IR spectrum reveals a hot component at $\sim 13 \mathrm{kK}$ and a cool one at $\sim 4.6 \mathrm{kK}$. The latter would suggest a late-type K2-K5 companion star, a distance range of 1.4-3.6 kpc, and an orbital period of 7-9 h. A near-UV variability ( $\approx 6 \mathrm{~h}$ ) also suggests a longer orbital period than previously estimated.

Conclusions. The analysis shows that the X-ray and UV/optical/near-IR emissions are more compatible with an accretion-powered compact object than with a rotational powered pulsar. The X-ray to UV bolometric luminosity ratio could be consistent with a binary hosting a neutron star, but the uncertainties in the radio data may also allow an LMXB black hole with a compact jet. In this case, it would be the first associated with a high-energy gamma-ray source.

Key words. accretion, accretion disks - X-rays: binaries - gamma rays: stars - binaries: close

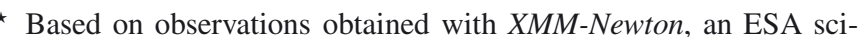
ence mission with instruments and contributions directly funded by ESA Member States and NASA, with Swift, a NASA science mission with Italian participation, with Rossi-XTE, a NASA science mission, and with Fermi a NASA mission with contributions from France, Germany, Italy, Japan, Sweden, and USA, and with AGILE, an Italian Space Agency mission with participation of the Italian Institute of Astrophysics and the Italian Institute of Nuclear Physics.
}

\section{Introduction}

XSS J12270-4859 (henceforth XSS J1227) is a peculiar and enigmatic hard X-ray source. An early proposal that it could be a cataclysmic variable (CV) hosting a magnetic white dwarf (Masetti et al. 2006; Butters et al. 2008) was disregarded with independent observations by Pretorius (2009), Saitou et al. (2009), and de Martino et al. (2010, hereafter 
dM10). These observations instead suggested a low-mass X-ray binary (LMXB) nature. A putative $4.32 \mathrm{~h}$ orbital period was also claimed from optical and possibly near IR photometry by dM10, but not confirmed by further near-IR (nIR) data (Saitou et al. 2011). In dM10 we discovered this source to be positionally associated to the bright unidentified gamma-ray Fermi-LAT source, 1FGL J1227.9-4852, detected up to $10 \mathrm{GeV}$. 1FGL J1227.9-4852 is also reported in the 2nd Fermi-LAT catalogue as 2FGL J1227.7-4853 (hereafter 2FGL J1227; Nolan et al. 2012). Based on this association, we found that the gamma-ray emission is a non-negligible fraction of the $\mathrm{X}$-ray luminosity $\left(L_{0.1-100 \mathrm{GeV}} / L_{0.2-100 \mathrm{keV}} \sim 0.8\right)$ and if the X-ray and gamma-ray emissions are linked, the peak energy should be between $1-100 \mathrm{MeV}$.

Due to its peculiar flaring and dipping behaviour, XSS J1227 was proposed to share some similarities, though with rather different timescales and energetics, with type-II burst sources and to represent an unusual low-luminosity $\left(L_{\mathrm{X}} \sim 6 \times 10^{33} \mathrm{erg} \mathrm{s}^{-1}\right.$ at $1 \mathrm{kpc}$ ) LMXB (dM10).

The nature of the compact object has been further investigated in other wavelength domains (Saitou et al. 2011; Hill et al. 2011). From radio searches conducted by Hill et al. (2011), three sources within the Fermi-LAT 99\% error box were detected, with only one having an obvious X-ray counterpart. This faint radio source is located at the position of XSS J1227 with flux densities of $0.18 \mathrm{mJy}$ at $5.5 \mathrm{GHz}$ and of $0.14 \mathrm{mJy}$ at $9 \mathrm{GHz}$. It was not detected at lower frequencies $(640 \mathrm{MHz}$, $240 \mathrm{MHz}$, and at $1400 \mathrm{MHz}$ ) in follow-up GMRT observations. For a power-law spectrum $S_{v} \sim v^{\alpha}$, the radio data gave a fairly unconstrained power-law index $\alpha=-0.5 \pm 0.6$. Also, Hill et al. (2011) did not detect fast radio pulses down to millisec. (msec) in subsequent Parkes observations. Nonetheless, the association with the Fermi-LAT source favoured XSS J1227 as a msec pulsar (MSP) binary (Hill et al. 2011), similar to the first discovered rotational-powered MSP in a quiescent LMXB PSR J1023+0038 (Tam et al. 2010). The number of MSP binaries detected in gamma-rays by Fermi-LAT has rapidly increased in the last year with the newly discovered systems PSR J12311411, PSR J0614-3329, and PSR J2214+3000 (Ransom et al. 2011), PSR J1816+4510 (Kaplan et al. 2012), PSR J0101-6422, PSR J1514-4946, and PSR J1902-5105 (Kerr et al. 2012). A few are found to host so-called black widow pulsars such as PSR J2051-0827(Wu et al. 2012) and J 2339+0533 (Romani \& Shaw 2011; Kong et al. 2012). A number of them are detected as faint X-ray sources with X-ray luminosities typical of radio MSPs (e.g. Ransom et al. 2011; Wu et al. 2012). Hence, it is expected that the Fermi satellite will discover most of the local population of MSPs, thus allowing constraint of the emission mechanisms and the binary evolution of their progenitors.

On the other hand, from the detection of simultaneous X-ray and nIR flares and the similarity of broad-band spectral energy distribution (SED) at low energies with black hole $(\mathrm{BH})$ candidates such as GRS 1915+105, XTE J1118+480 and Cyg X-3, Saitou et al. (2011) proposed that XSS J1227 is reminiscent of microquasars with a synchrotron jet. However, broad-band SEDs, if due to synchrotron cooling in optically thin regime, are expected to show a break at $\sim 500 \mathrm{keV}$, making them undetectable in the high-energy gamma-rays. The recent Fermi and AGILE detections of high-mass X-ray binaries (HMXBs; Abdo et al. 2009c; Tavani et al. 2009b; Ackermann et al. 2012; Hadasch et al. 2012), with some of them still thought to host neutron stars (NS) or BH (Hadasch et al. 2012; Papitto et al. 2012; Torres et al. 2012) may open the possibility of detecting faint gamma-ray sources in different binary types (Hadasch et al. 2012).

To further advance our understanding of this intriguing object, we started a X-ray monitoring campaign in 2011 to infer whether this source undergoes changes in luminosity and to detect possible fast $(\mathrm{msec})$ pulsations in the X-ray band. We were also granted AGILE/GRID data acquired since the beginning of operations in 2007 to detect for the first time this source in the soft gamma rays down to $50 \mathrm{MeV}$ to obtain a broad-band gamma-ray coverage combining the GRID and LAT data. With these aims, we present the X-ray observations acquired along one year with Swift and Rossi-XTE (RXTE) and the fast timing X-ray data acquired with XMM-Newton. In Sect. 2 we report the observations and data reduction; in Sect. 3 we provide analysis of the X-ray light curves, including the long-term comparison with the gamma-ray Fermi-LAT curve and the search for fast msec pulses; in Sect. 4 we examine the spectral properties over a wide energy range, and in Sect. 5, we discuss the possible nature of this object.

\section{Observations and data reduction}

The new observations acquired with XMM-Newton, RXTE, and Swift as well as the time coverage of AGILE/GRID are summarised in Table 1.

\subsection{The XMM-Newton observation}

A $30 \mathrm{ks}$ pointing with XMM-Newton (OBSID: 656780901) was carried out on Jan.1, 2011. In contrast to our past observation in 2009 (dM10), the EPIC-pn camera (Strüder et al. 2001) was operated in the timing read-out mode that reaches a resolution of $0.03 \mathrm{~ms}$. The EPIC-MOS cameras (Turner et al. 2001) were instead operated in imaging full-window mode using the thin filters. The optical monitor (OM; Mason et al. 2001) was operated in fast-window mode using the U (3000-3800 $\AA$ ) filter throughout the observation (see Table 1). The data were processed using the standard reduction pipelines and analysed with the SAS 10.0 package using the latest calibration files. The photon arrival times from EPIC cameras and OM were reported to the solar system barycentre using the nominal position of XSS J1227 (Masetti et al. 2006). In the timing-mode read-out used for the EPIC-pn exposure, the spatial information is lost because imaging is made only in one dimension and the data from a predefined area on one CCD chip are collapsed into a one-dimensional row to be read out at high speed. The EPIC-pn events from the source and background were extracted from RAWX $=24-52 \mathrm{px}$ and from RAWX $=3-15 \mathrm{px}$, respectively. For the EPIC-MOS cameras, we instead extracted events using a circular region of $37^{\prime \prime}$ aperture radius centred on the source and using a background region located on the same $\mathrm{CCD}$ chip. In order to improve the signal-to-noise ratio $(\mathrm{S} / \mathrm{N})$, we filtered the data by selecting pattern pixel events up to double with zero-quality flag for the EPIC-pn data, and up to quadruple pixel events for the EPICMOS data. The average background level of the EPIC cameras was low during the whole observation. The backgroundsubtracted OM-U light curve was obtained with a binning time of $10 \mathrm{~s}$.

\subsection{The RXTE observations}

In order to infer the mid- to long-term behaviour of XSS J1227, we initiated a monitoring with RXTE (Bradt et al. 1993, 
Table 1. Summary of the observations of XSS J1227.

\begin{tabular}{|c|c|c|c|c|c|}
\hline $\begin{array}{l}\text { Telescope } \\
\text { Prog. ID }\end{array}$ & Instrument & Date & UT (start) & Exposure time (s) & Net count rate (counts s${ }^{-1}$ ) \\
\hline $\begin{array}{l}\text { XMM-Newton } \\
65678\end{array}$ & $\begin{array}{c}\text { EPIC-pn } \\
\text { EPIC-MOS } \\
\text { OM-U }\end{array}$ & 2011-01-01 & $\begin{array}{l}07: 06 \\
06: 47 \\
06: 56 \\
07: 46 \\
09: 07 \\
09: 57 \\
10: 48 \\
11: 38 \\
12: 25 \\
13: 12 \\
13: 59 \\
14: 46\end{array}$ & $\begin{array}{c}30045 \\
31022 \\
2699 \\
2700 \\
2700 \\
2699 \\
2700 \\
2499 \\
2499 \\
2499 \\
2498 \\
2500\end{array}$ & $\begin{array}{c}3.71 \pm 0.09 \\
1.09 \pm 0.01 \\
5.19 \pm 0.04\end{array}$ \\
\hline $\begin{array}{l}\text { RXTE }^{5} \\
96309\end{array}$ & PCA & $\begin{array}{l}2011-01-03 \\
2011-01-10 \\
2011-01-16 \\
2011-01-23 \\
2011-01-31 \\
2011-02-07 \\
2011-02-14 \\
2011-02-21 \\
2011-02-28 \\
2011-03-07 \\
2011-03-14 \\
2011-03-21 \\
2011-03-28 \\
2011-04-03 \\
2011-04-13 \\
2011-04-18 \\
2011-04-25 \\
2011-05-03 \\
2011-05-10 \\
2011-05-16 \\
2011-05-23 \\
2011-05-30 \\
2011-06-06 \\
2011-06-13 \\
2011-06-19 \\
2011-06-27 \\
2011-07-04 \\
2011-07-11 \\
2011-07-18 \\
2011-07-25 \\
2011-08-01 \\
2011-08-08 \\
2011-08-15 \\
2011-08-22 \\
2011-08-29 \\
2011-09-19 \\
2011-09-27 \\
2011-10-08 \\
2011-10-21 \\
2011-11-10 \\
2011-11-20 \\
2011-11-30 \\
2011-12-10 \\
2011-12-23\end{array}$ & $\begin{array}{l}00: 27 \\
14: 11 \\
17: 38 \\
12: 37 \\
13: 26 \\
13: 38 \\
15: 05 \\
17: 49 \\
12: 51 \\
10: 58 \\
05: 59 \\
12: 32 \\
21: 22 \\
18: 31 \\
09: 03 \\
14: 18 \\
06: 12 \\
19: 58 \\
21: 05 \\
13: 17 \\
17: 37 \\
15: 49 \\
06: 14 \\
02: 34 \\
04: 14 \\
17: 23 \\
14: 18 \\
10: 34 \\
13: 33 \\
11: 39 \\
03: 25 \\
06: 32 \\
06: 00 \\
08: 38 \\
17: 41 \\
21: 22 \\
04: 51 \\
12: 01 \\
20: 53 \\
18: 09 \\
14: 50 \\
18: 27 \\
00: 03 \\
02: 12\end{array}$ & $\begin{array}{l}2013 \\
2000 \\
1917 \\
2082 \\
1919 \\
2052 \\
1947 \\
1836 \\
2476 \\
1977 \\
2043 \\
1962 \\
1874 \\
1794 \\
1919 \\
1770 \\
1962 \\
1586 \\
1922 \\
1909 \\
2010 \\
2034 \\
1742 \\
1807 \\
2043 \\
2099 \\
1824 \\
1789 \\
1965 \\
1716 \\
1947 \\
2102 \\
2004 \\
1756 \\
2132 \\
1859 \\
1656 \\
1731 \\
2025 \\
1933 \\
1860 \\
2094 \\
1787 \\
1947 \\
\end{array}$ & $\begin{array}{l}1.72 \pm 0.06 \\
1.83 \pm 0.06 \\
1.94 \pm 0.05 \\
2.21 \pm 0.07 \\
2.03 \pm 0.07 \\
0.96 \pm 0.06 \\
1.84 \pm 0.06 \\
0.97 \pm 0.06 \\
1.59 \pm 0.06 \\
2.44 \pm 0.07 \\
2.05 \pm 0.07 \\
0.89 \pm 0.06 \\
0.56 \pm 0.06 \\
1.07 \pm 0.06 \\
1.73 \pm 0.06 \\
2.25 \pm 0.07 \\
1.90 \pm 0.06 \\
1.05 \pm 0.08 \\
1.82 \pm 0.07 \\
1.03 \pm 0.06 \\
1.63 \pm 0.06 \\
0.91 \pm 0.06 \\
2.06 \pm 0.07 \\
1.09 \pm 0.06 \\
2.27 \pm 0.07 \\
1.83 \pm 0.07 \\
1.34 \pm 0.06 \\
1.06 \pm 0.07 \\
1.51 \pm 0.07 \\
1.51 \pm 0.06 \\
2.06 \pm 0.07 \\
1.94 \pm 0.06 \\
0.84 \pm 0.06 \\
0.90 \pm 0.07 \\
1.61 \pm 0.06 \\
1.10 \pm 0.06 \\
0.83 \pm 0.06 \\
1.03 \pm 0.07 \\
1.03 \pm 0.06 \\
2.24 \pm 0.07 \\
0.80 \pm 0.06 \\
0.84 \pm 0.07 \\
0.84 \pm 0.06 \\
1.04 \pm 0.06\end{array}$ \\
\hline
\end{tabular}

Notes. ${ }^{(4)}$ Total EPIC count rates in the $0.2-10 \mathrm{keV}$ range ${ }^{(5)}$ Total RXTE/PCA count rates in the $2-9 \mathrm{keV}$ range. ${ }^{(6)}$ Total Swift/XRT count rates in the $0.3-10 \mathrm{keV}$ range. ${ }^{(7)}$ AGILE/GRID Pointing and spinning mode data: the start and end time and total exposure are reported. 
Table 1. continued.

\begin{tabular}{|c|c|c|c|c|c|}
\hline $\begin{array}{l}\text { Telescope } \\
\text { Prog. ID }\end{array}$ & Instrument & Date & UT (start) & Exposure time (s) & Net count rate (counts s ${ }^{-1}$ ) \\
\hline Swift ${ }^{6}$ & XRT & $2011-03-23$ & $04: 35$ & 672 & $0.17 \pm 0.09$ \\
\hline \multirow[t]{18}{*}{41135} & & $2011-03-23$ & $06: 11$ & 732 & $0.26 \pm 0.11$ \\
\hline & & 2011-03-23 & $07: 47$ & 701 & $0.26 \pm 0.09$ \\
\hline & & 2011-03-23 & 09:24 & 729 & $0.28 \pm 0.12$ \\
\hline & & $2011-03-23$ & 11:00 & 729 & $0.35 \pm 0.12$ \\
\hline & & 2011-03-23 & $12: 36$ & 852 & $0.27 \pm 0.09$ \\
\hline & & $2011-03-23$ & $14: 13$ & 792 & $0.30 \pm 0.09$ \\
\hline & & 2011-05-10 & $16: 32$ & 634 & $0.67 \pm 0.15$ \\
\hline & & 2011-09-15 & $07: 55$ & 854 & $0.32 \pm 0.13$ \\
\hline & & 2011-09-18 & 09:48 & 809 & $0.07 \pm 0.06$ \\
\hline & & 2011-09-19 & 04:59 & 1483 & $0.39 \pm 0.15$ \\
\hline & & 2011-09-19 & $06: 34$ & 859 & $0.17 \pm 0.09$ \\
\hline & & 2011-09-19 & $08: 12$ & 802 & $0.22 \pm 0.10$ \\
\hline & & 2011-09-19 & $22: 39$ & 392 & $0.34 \pm 0.14$ \\
\hline & & 2011-09-21 & 02:02 & 654 & $0.31 \pm 0.13$ \\
\hline & & 2011-09-21 & 10:11 & 242 & $0.15 \pm 0.08$ \\
\hline & & 2011-09-22 & $21: 16$ & 557 & $0.28 \pm 0.12$ \\
\hline & & $2011-09-25$ & $02: 11$ & 1293 & $0.39 \pm 0.15$ \\
\hline & & 2011-09-26 & $02: 20$ & 557 & $0.24 \pm 0.09$ \\
\hline AGILE $^{7}$ & GRID & 2007-10-01 & $2009-10-31$ & $5.3 \times 10^{6}$ & \\
\hline 70 & & $2009-11-15$ & 2011-05-15 & $3.8 \times 10^{6}$ & \\
\hline
\end{tabular}

Prog.ID: 96309) that started in Jan. 2011 and ended in Dec. 2011. A total of $44 \mathrm{RXTE} / \mathrm{PCA}$ pointings were performed with a cadence of about one week and typical exposure times of $2 \mathrm{ks}$ (see Table 1). The RXTE/PCA standard data products for each observations were obtained from the RXTE Guest Observer facility. Barycentric corrections were applied to the backgroundsubtracted light curves. XSS J1227 was typically found at a net count rate of $\sim 0.3-0.5 \mathrm{cts} \mathrm{s}^{-1} / \mathrm{PCU}$ in the $9-20 \mathrm{keV}$. Due to the poor statistics above $10 \mathrm{keV}$, the timing analysis is restricted to the $2-9 \mathrm{keV}$ range, also using the soft $2-4 \mathrm{keV}$ and hard 4-9 keV bands. The source spectra were instead analysed up to $30 \mathrm{keV}$ in each observation.

\subsection{The Swift observations}

The monitoring of XSS J1227 with Swift/XRT (Prog.ID:41135) started in Mar. 2011 and ended in Sept. 2011. A total of 19 snapshots were performed with typical exposure times of $800 \mathrm{~s}$. A denser coverage was performed on Mar. 23 and on Sept. 19 (see Table 1). We used the Swift/XRT data products generator at the University of Leicester (Evans et al. 2009) to build backgroundsubtracted light curves in the $0.3-10 \mathrm{keV}, 0.3-1.5 \mathrm{keV}$, and $1.5-10 \mathrm{keV}$ bands and spectra in the $0.3-10 \mathrm{keV}$ range. We used photon counting-mode data only. Barycentric corrections were applied to the extracted light curves.

\subsection{The AGILE data}

Astrorivelatore Gamma ad Immagini LEggero (AGILE; Tavani et al. 2009a) consists of a large field of view $\left(\sim 60^{\circ}\right)$ gamma-ray imager, GRID, sensitive in the energy range $30 \mathrm{MeV}-50 \mathrm{GeV}$, and a co-axial hard X-ray detector (Super-AGILE) for imaging in the 18-60 keV range (Feroci et al. 2007). Since beginning operation in Jul. 2007, AGILE has worked in pointing mode, but science operations were reconfigured following a malfunction of the rotation wheel in mid-Oct. 2009. Since then, the satellite has been operating in "spinning observing mode", with the solar panels pointing at the Sun and the instrument axis sweeping the sky with an angular speed $\sim 1 \mathrm{deg} \mathrm{s}^{-1}$. After our association of XSS J1227 to the Fermi source (dM10), we were granted AGILE/GRID data in Cycle 3 (Prog.ID:70). We have enlarged the dataset including observations collected in pointing mode from Oct. 1, 2007 to Oct. 31, 2009, and the data collected with the satellite in spinning mode from Oct. 31, 2009 to May 15, 2011; the total exposure times amount to $5.3 \mathrm{Ms}$ and $3.8 \mathrm{Ms}$ respectively.

XSS J1227 is too faint in the X-rays to be detected by the Super-AGILE instrument and therefore only the GRID dataset was analysed using the Build 20 release of the GRID analysis software available at the ASI-Science Data Centre (SDC). Well-reconstructed $\gamma$-ray events were selected using the FM3.119 filter. Events collected during the passage in the South Atlantic Anomaly and Earth-albedo gamma rays coming from a circular region of radius $80^{\circ}$ centred on the Earth were rejected. A maximum likelihood analysis was performed in the $100 \mathrm{MeV}-50 \mathrm{GeV}$ energy band, obtaining $2 \sigma$ upper limits of $7 \times 10^{-8}$ photons $\mathrm{cm}^{-2} \mathrm{~s}^{-1}$ with a total exposure coverage of $2 \times 10^{9} \mathrm{~cm}^{2} \mathrm{~s}$ for the pointing-mode period and $1.2 \times 10^{-7}$ photons $\mathrm{cm}^{-2} \mathrm{~s}^{-1}$ with a total exposure coverage of $1.5 \times 10^{9} \mathrm{~cm}^{2} \mathrm{~s}$ for the spinning-mode period. For the total period under analysis we obtained a $2 \sigma$ upper limit of $6 \times 10^{-8}$ photons $\mathrm{cm}^{-2} \mathrm{~s}^{-1}$ with a total exposure coverage of 
$\sim 3.4 \times 10^{9} \mathrm{~cm}^{2} \mathrm{~s}$. In order to investigate soft $(E<200 \mathrm{MeV})$ gamma-ray emission from XSS J1227, we also integrated the GRID data in the non-standard 50-200 MeV energy band, obtaining a $2 \sigma$ upper limit of $14.8 \times 10^{-8}$ photons $\mathrm{cm}^{-2} \mathrm{~s}^{-1}$ with a total exposure coverage of $2 \times 10^{9} \mathrm{~cm}^{2} \mathrm{~s}$.

\subsection{The Fermi data}

The Fermi-LAT data were retrieved from the Fermi Science Support Centre. The dataset spans 44 months since the start of Fermi operations, i.e. from Aug. 4, 2008 to Apr. 17,2012, amounting to a total exposure time of 52.6 Ms. We used Pass7 photon data, reducing and analysing them using the Fermi Science Tools v9r27 package. We used the high-quality (diffuse) photon event class (EVENT CLASS =2) and the Pass 7 v6 Source (P7 V6 source) instrument response functions (IRFs). We excluded time periods when the region around 2FGL J1227 was observed at a zenith angle greater than $105^{\circ}$ to reduce contamination by Earth albedo gamma rays. Correction to the solar system baricentre was also applied.

We produced a LAT aperture photometry light curve with a time bin of four days from a circular region centred on the source position and a radius of $1^{\circ}$ to avoid contamination from close sources (2FGL J1218.8-4827, 2FGL J1231.3-5112 and 2FGL J1207.3-5055) (see Nolan et al. 2012). We performed this operation with the gtbin task and applied the exposure correction using the gtexposure task. These tasks do not perform background subtraction, and hence no background correction was applied.

The spectral fluxes in the five LAT bands $(100-300 \mathrm{MeV}$, $0.3-1 \mathrm{GeV}, 1-3 \mathrm{GeV}, 3-10 \mathrm{GeV}$, and $10-100 \mathrm{GeV}$ ) were instead collected from the 2 nd source catalogue that covers 24 months of operations and were obtained using maximum likelihood analysis (see details in Nolan et al. 2012). 2FGL J1227 is reported at a flux of $3.34 \pm 0.23 \times 10^{-11} \mathrm{erg} \mathrm{cm}^{-2} \mathrm{~s}^{-1}$ in the $0.1-100 \mathrm{GeV}$ range at a significance of $24.3 \sigma$ in accordance with the value of $3.95 \pm 0.44 \times 10^{-11} \mathrm{erg} \mathrm{cm}^{-2} \mathrm{~s}^{-1}$ reported in the $1 \mathrm{st}$ LAT source catalogue. The localization procedure used to construct the catalogue also provides spectral fits to all sources. In contrast to the 1 st catalogue, which used only a power-law function, different spectral shapes are used in the 2nd one. For 2FGL J1227, a power-law and a function, called "logParabola" are fitted to the spectrum. This representation, allows for a smoother decrease at high energy than a power-law exponential cutoff form. It reduces to a simple power-law when the curvature parameter $\beta=0$ and is reported in the catalogue when the curvature is significant by above $4 \sigma$ (Nolan et al. 2012). A simple power-law fit is reported with a spectral index of 2.3306. The spectral index of the $\log$ Parabola is $2.085 \pm 0.087, \beta=0.288 \pm 0.064$ with a pivot energy at $549.30 \mathrm{MeV}$ (where the uncertainty on differential flux is minimal). The significance of the fit improvement with respect to the simple power-law is $5.6 \sigma$.

\section{Results}

\subsection{X-ray persistent characteristics}

The background-subtracted X-ray light curves from EPIC-pn and MOS cameras were first extracted in $60 \mathrm{~s}$ bins to evaluate the overall behaviour of XSS J1227 during the XMM-Newton observation. The light curves from the EPIC-pn and MOS are similar, and we use the EPIC-pn data because of the higher S/N. In Fig. 1, the $0.3-10 \mathrm{keV}$ light curve with a binning time of $10 \mathrm{~s}$ is shown together with the temporal behaviour of the hardness ratio $\mathrm{HR}=[\mathrm{H}-\mathrm{S} / \mathrm{H}+\mathrm{S}]$, where $\mathrm{S}$ and $\mathrm{H}$ are the count rates in the $0.3-2 \mathrm{keV}$ and $2-10 \mathrm{keV}$, respectively, with a binning time of $60 \mathrm{~s}$. XSS J1227 was found to be at a persistent (quiescent) level of $\sim 4.35 \pm 0.35 \mathrm{cts} \mathrm{s}^{-1}$ for most of the EPIC-pn exposure, except for the last $4 \mathrm{ks}$, where the count rate increases up to a factor of 6.4. While the general behaviour observed in 2011 is similar to that of the Jan. 2009 XMM-Newton observation (dM10), the EPIC-pn timing mode data show that the persistent level is characterized by dips of variable length (see Sect. 3.3).

The hardness ratios confirm the general trend observed in 2009 (dM10), where a substantial hardening is detected during the postflare dips and there is no major spectral change during flares and quiescent dips. The intensity-versus-hardness ratio diagram (HID) in Fig. 2 is similar (see Fig. 4 of dM10). Here we define flares when the count rate $>5.7 \mathrm{cts} \mathrm{s}^{-1}$ (cyan in Fig. 2) and dips have a count rate $<3$. $\operatorname{cts~s}^{-1}$. The higher $\mathrm{S} / \mathrm{N}$ EPIC-pn data allow the dips to be better sampled. Therefore we further separate soft dips with $\mathrm{HR}<-0.6$ (blue) and hard dips with $\mathrm{HR}>-0.2$ (red) from "ordinary" neutral dips with $-0.6<\mathrm{HR}<-0.2$ (magenta). The hard and soft dips are defined as those below/above $2 \sigma$ the average HR value in quiescence. The HID confirms that only post-flare dips are hard, while dips during quiescence are mostly neutral with a marginal softening in those that are longer.

Flares and dips in the X-ray light curve of XSS J1227 are found in all observations conducted in 2011 by RXTE and Swift. In Fig. 3, we show the light curves of each RXTE pointing with a binning time of $16 \mathrm{~s}$. The hardness ratios $\mathrm{HR}_{\mathrm{RXTE}}=$ $[\mathrm{H}-\mathrm{M} / \mathrm{H}+\mathrm{M}]$ in the $2-4 \mathrm{keV}$ and the $4-9 \mathrm{keV}$ bands were constructed with a binning time of $128 \mathrm{~s}$. We define the quiescent level as that observed on Dec. 23 (Table 1), and hence flares are defined with a count rate $>1.8 \mathrm{cts} \mathrm{s}^{-1}$ and dips with a count rate $<0.8 \mathrm{cts} \mathrm{s}^{-1}$. The average hardness ratio in quiescence is $\mathrm{HR}_{\mathrm{RXTE}}=0.38 \pm 0.24$, and we define hard/soft dips as those over/below $2 \sigma$ this average value, respectively. Ordinary neutral dips are instead those found within this range. The RXTE HID (Fig. 4) shows that flares and ordinary and soft dips have been relatively well sampled, but not the hard dips. This is likely due to the higher energy coverage of RXTE/PCA with respect to XMM-Newton and Swift instruments.

The Swift light curves (not shown) were extracted with a binning time of $25 \mathrm{~s}$. Again, XSS J1227 is found stable at $\sim 0.2-0.3 \mathrm{cts} \mathrm{s}^{-1}$ during most of Swift pointings (see Table 1). Despite the poor coverage, several flares are detected or partially detected, with one notably on Sept. 19 that reached peak intensity $\sim 8$ times the quiescent $\left(0.26\right.$ cts s$\left.^{-1}\right)$ level. The flares are defined with a count rate $>0.43 \mathrm{cts} \mathrm{s}^{-1}$, while dips are defined with a count rate $<0.2 \mathrm{cts} \mathrm{s}^{-1}$. The hardness ratios were constructed with a binning time of $128 \mathrm{~s}$ in the $0.2-1.5 \mathrm{keV}$ and $1.5-10 \mathrm{keV}$ bands. Soft/hard dips were defined as over/below $2 \sigma$ the average value $\mathrm{HR}_{\text {Swift }}=0.1 \pm 0.1$. The HID from Swift also reveals the tendency of hardening during post-flare dips (Fig. 5).

We also inspected archival Swift unpublished observations on Sept. 15, 2005, Sept. 24, 2005 and Aug. 10, 2010 in similar fashion as the 2011 Swift pointings to construct a long-term history of XSS J1227.

The data acquired during the 2011 monitoring compared with the observations carried out in 2005 by Swift, in 2007 by RXTE (dM10), in 2008 by Suzaku (Saitou et al. 2009), in 2009 with XMM-Newton (dM10) and RXTE (Saitou et al. 2011), and in 2010 by Swift allow to definitively assess that XSS J1227 is a persistent and not a transient source. It is stable on a 7-yr timescale while undergoing repetitive flaring episodes. From the longer datasets we also estimate flare occurrence and 


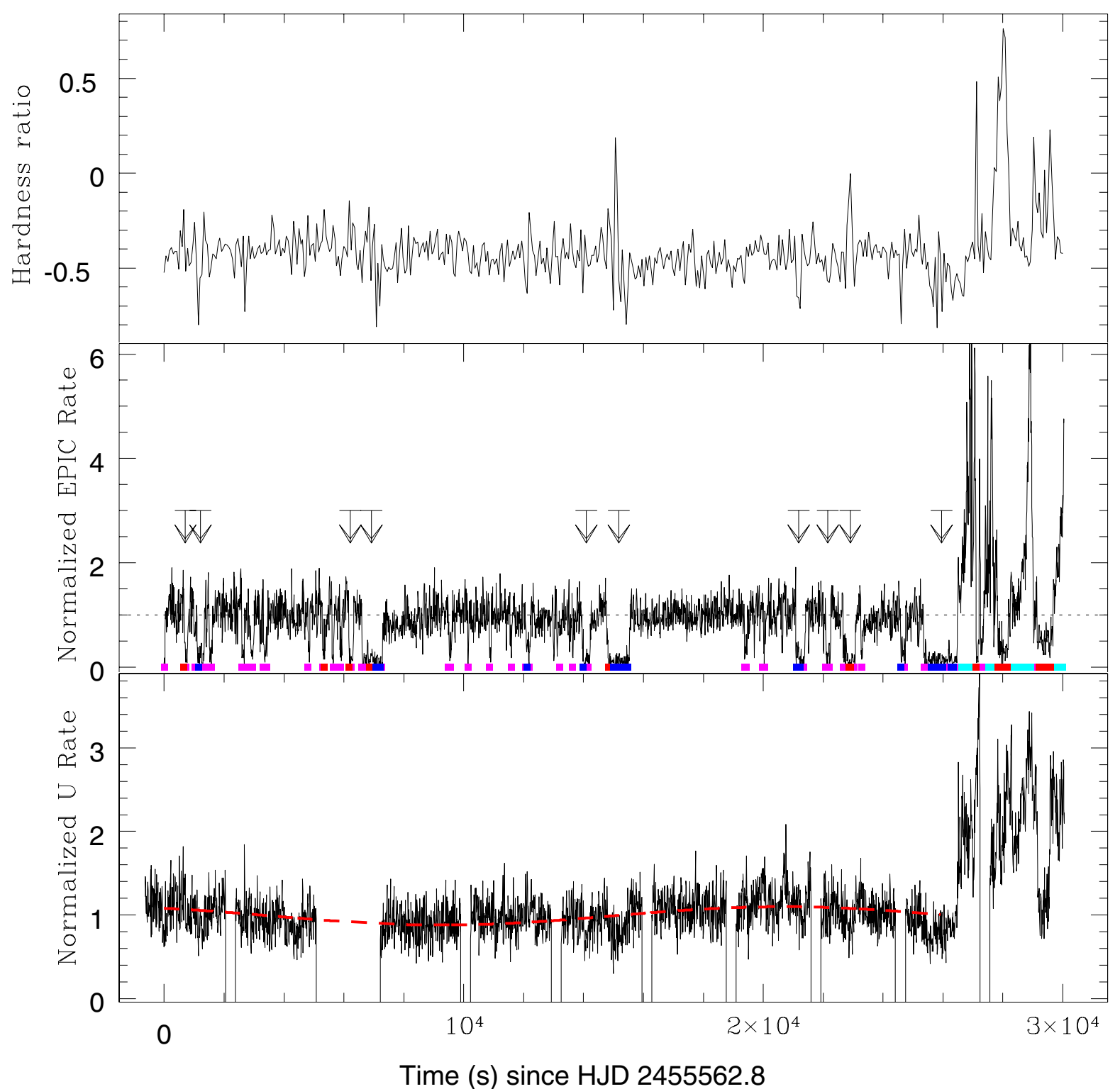

Fig. 1. Bottom panel: net $U$ band light curve with a $10 \mathrm{~s}$ binning time. Count rates are normalized to the average persistent quiescence value. A sinusoidal fit with a period of $6.4 \mathrm{~h}$ is also reported. Gaps are due to the OM fast acquisition mode. Middle panel: the EPIC-pn net light curve in the $0.3-10 \mathrm{keV}$ range with a $10 \mathrm{~s}$ binning time. The count rate is normalized to the average quiescent value. Flares are marked in cyan, dips with no spectral change in magenta, dips showing a softening are marked in blue, while hard dips are marked in red. Arrows mark ten long dips used to study the CCF and spectra. Top panel: the hardness ratio between the $0.3-2 \mathrm{keV}$ and $2-10 \mathrm{keV}$ bands with binning time of $60 \mathrm{~s}$. (See electronic edition for colour version.)

duration: $\Delta_{\text {Quie }} \gtrsim 24 \mathrm{ks}$ and $\Delta_{\text {Flares }} \gtrsim 8.2 \mathrm{ks}$ from Suzaku (2008); $\Delta_{\text {Quie }} \gtrsim 35 \mathrm{ks}$ and $\Delta_{\text {Flares }} \gtrsim 3.6 \mathrm{ks}$ from RXTE (2007) and $\Delta_{\text {Quie }}=27.1 \mathrm{ks}$ and $\Delta_{\text {Flares }} \gtrsim 3.1 \mathrm{ks}$ from XMM-Newton (2009) and $\Delta_{\text {Quie }} \gtrsim 27.4 \mathrm{ks}$ and $\Delta_{\text {Flares }} \gtrsim 3.4 \mathrm{ks}$ from XMM-Newton (2011), giving lower limits of $\Delta_{\text {Quie }}=27-35 \mathrm{ks}$ and $\Delta_{\text {Flares }} \sim$ $4 \mathrm{ks}$, the latter grouped in multi-flares. These could be a signature of $\mathrm{a} \gtrsim 9-11 \mathrm{~h}$ occurrence.

\subsection{The near-UV light}

Figure 1 also reports the simultaneous light curve recorded with the $\mathrm{OM}$ in the $U$ band filter in Jan.2011, where the source also undergoes a flaring activity at the end of the XMM-Newton observation. XSS J1227 is at an average $U$ band magnitude of 16.6 mag during quiescence and reaches $15.1 \mathrm{mag}$ at flares.
The highest peak in the $U$ light curve is $\sim 3$ times the persistent level, smaller than the X-ray variations. Despite the gaps in this light curve, it is apparent that dips occur also in the near-UV during quiescence, with the count rate dropping by a factor of 1.4. Hence, in contrast to what was claimed in dM10, it is now possible to identify the UV counterparts of the X-ray dips in XSS J1227. An enlargement of the light curves is shown in Fig. 6.

Furthermore, a long-term variation in the UV was suspected to be present in the 2009 XMM-Newton OM data. A Scargle periodogram of the quiescent $U$ band time series shows a peak at $6.7 \mathrm{~h}$ (at $3.6 \sigma$ c.1.). A formal sinusoidal fit gives a period of $6.4 \pm 0.2 \mathrm{~h}$ (uncertainty at $1 \sigma$ c.l.) and full amplitude of $22 \pm 1 \%$ (see also Fig. 1). The statistical significance of the inclusion of a sinusoid with respect to a constant function, evaluated with a F-test, is $10 \sigma$. Given that the length of the $U$ band exposure 


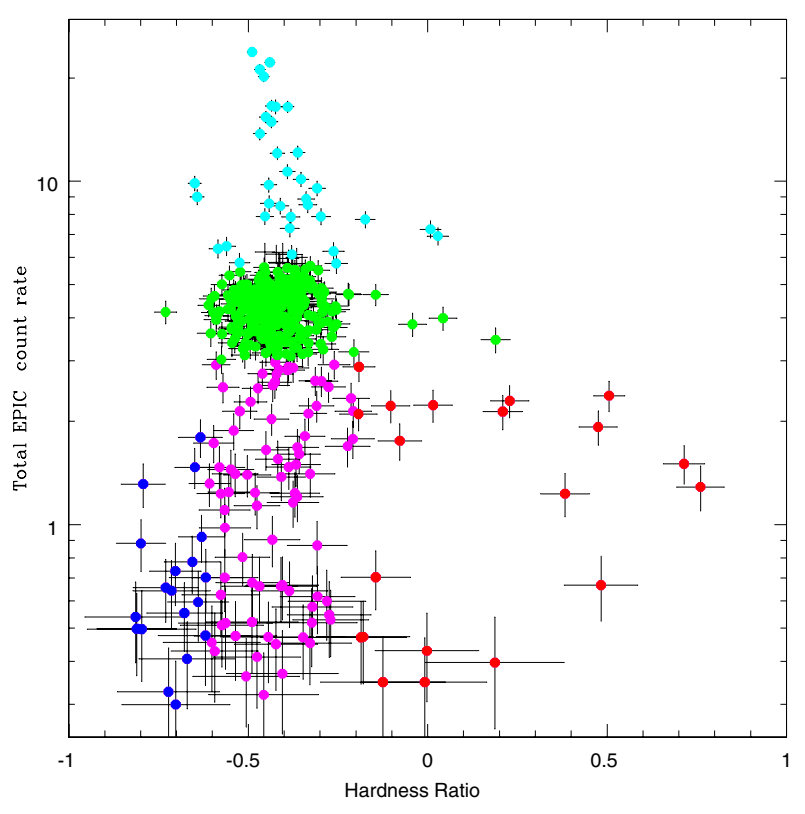

Fig. 2. HID between $0.3-2 \mathrm{keV}$ and $2-10 \mathrm{keV}$ EPIC bands. Flares are marked in cyan, dips with no spectral change in magenta, soft dips in blue, and hard dips in red. Green points represent quiescence. (See electronic version for colour figure.)

during quiescence is $26 \mathrm{ks}$, the $6.4 \mathrm{~h}$ period should be regarded as a lower limit. We note here that, in contrast to what was claimed from the past XMM-Newton pointing (dM10), no variations on timescales of hours are found in this EPIC-pn quiescent light curve.

\subsection{The dips in focus}

The X-ray dips represent a persistent characteristic of XSS J1227 during quiescence. The time spent in the dips amounts to $7.78 \mathrm{ks}$, representing $\sim 30 \%$ of quiescent period. A few (ten are marked in Fig. 1) longer dips have durations between $100 \mathrm{~s}$ and $700 \mathrm{~s}$, but the one observed prior the flaring activity is the longest, with a duration of 1100 s. Similarly, in the 2009 XMM-Newton pointing, the longest dip is detected before the flaring activity. In both observations, these dips are soft. The source also undergoes several short dips of length 10-90 s, where the count rate does not drop to zero. These short dips are the ordinary neutral dips with no substantial spectral change (Fig. 2).

The selected ten long dips are characterised by a less steep, though rapid, decay reaching almost zero counts in the longer ones and a faster recovery to the persistent level. The decay and rise times have lengths ranging between $\sim 40-90 \mathrm{~s}$ and appear to be correlated with the length of the dips, with slower decays/rises in the longer dips. The hardness ratios show a softening only in the longer dips. We also performed a cross correlation (CCF) between the hard $(2-10 \mathrm{keV})$ and soft $(0.3-2 \mathrm{keV})$ light curves during the ten selected long dips. The CCF is shown in the righthand lower panel of Fig. 7, where the soft band is taken as a reference and its autocorrelation function (ACF) is also reported. The CCF does not reveal any lag between the two bands, implying that the bulk of X-ray flux arises from the same region.

Near-UV dips seen in quiescence occur almost simultaneously with the X-ray dips, but the flux deficiency is $\sim 40 \%$, hence much lower than their X-ray counterparts (Fig. 6). The shape of UV dips is also different, being shallower with a smooth decay and rise. This is reflected in the count rate ratios between the two bands. Using the total X-ray range as reference, we computed its ACF and cross-correlated the $U$ band light curve with the $\mathrm{X}$-ray one using eight of the ten dips covered simultaneously (see Fig. 1). The CCF is shown in the right-hand top panel of Fig. 7 together with the ACF of the X-ray band. It is peaked at zero lags, but an excess at negative lags $\sim 100-300 \mathrm{~s}$ is found. Both the UV deficiency and CCFs indicate that the UV-emitting region is more extended.

\subsection{The flaring states}

XSS J1227 showed flares at the end of the XMM-Newton observation where the $\mathrm{X}$-ray count rate increased by a factor of $\sim 6.4$ (Fig. 8). Similar to the 2009 XMM-Newton light curve, the flaring activity consists of multiple events. Here we identify at least four major events, lasting about $600 \mathrm{~s}, 640 \mathrm{~s}, 847 \mathrm{~s}$, and $>350 \mathrm{~s}$ (the latter covered only at the start). The higher S/N EPIC-pn timing mode data, while confirming the general exponential rise and rapid decay of major flares, also allow us to identify that they are highly structured and composed of multiple peaks with a typical timescale of 30-50 s. The major spectral changes occur during the dips after these flares, as depicted in Fig. 1, where hardening is detected but not at flare rise and maximum. We cross-correlated the hard $(2-10 \mathrm{keV})$ with the soft $(0.3-2 \mathrm{keV}$, taken as reference) bands and computed the ACF in the soft band in the flare portions during the rise and maximum. The CCF and ACF are shown in Fig. 7, where no delay is inferred, confirming what was found in 2009.

The flares, simultaneously detected in the $U$ band, display peak intensities up to a factor of $\sim 3$. In the $U$ band, only one post-flare dip can be recognized precisely after the third flare (Fig. 8). The CCF between $U$ and X-ray (taken as reference) peaks at zero lags but is also asymmetric towards negative lags $(\sim 150-200 \mathrm{~s})$. It is different from what was found in 2009, possibly because of the short coverage between the two bands in that observation. This could be due to the rather different structure of $\mathrm{U}$ flares with respect to the $\mathrm{X}$-ray ones, where not all post-flare X-ray dips have their UV counterpart and the last UV flare rises faster than the X-ray one.

\subsection{Search for fast coherent signals}

The EPIC-pn timing mode data were also inspected to search for fast periodic or quasi-periodic signals in the range $0.5-10 \mathrm{keV}$. In order to assess the presence of a coherent signal in the EPICpn observation of XSS J1227, a Fourier analysis was first performed using the whole exposure, binning the time series at eight times the temporal resolution of the EPIC-pn timing mode data, so that $t_{\text {bin }} \simeq 0.236 \mathrm{~ms}$. Hence the search for periodic signals was performed in the frequency interval $3.3 \times 10^{-5}-2114 \mathrm{~Hz}$. We took into account the number of frequency bins searched to define a threshold power level to be exceeded in the presence of a signal above the noise (see, e.g. van der Klis 1989). Because of the presence of red noise at low frequencies (Fig. 9), we used a smoothing window technique to evaluate the detection level at each of the frequencies searched (Israel \& Stella 1996). No signal was significantly detected at a confidence level of $3 \sigma$. Given the maximum power detected in the power spectrum, we evaluated an upper limit at $3 \sigma$ confidence level on the fractional amplitude of pulsations of $A_{\mathrm{UL}}^{\text {is }}=0.050$. The effects of the observation length and binning, as well as the interaction between a putative signal and noise, are accounted for as described by Vaughan et al. (1994). The power spectrum continuum below $100 \mathrm{~Hz}$ is shown in Fig. 9. Three flat-top noise components 

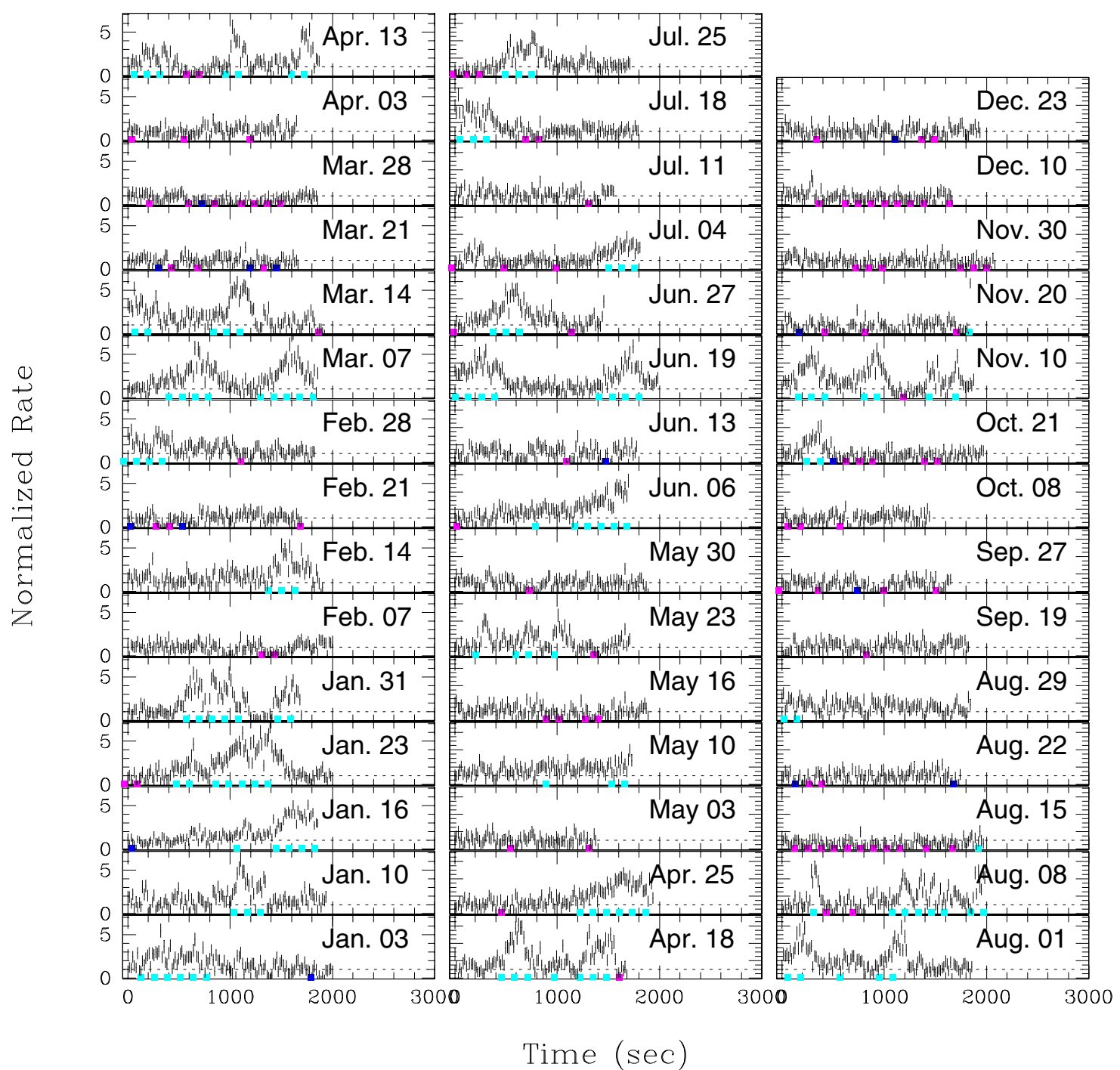

Time (sec)

Fig. 3. RXTE net light curves during the pointings in 2011 with a binning time of $16 \mathrm{~s}$. The count rate is normalized to the quiescent average value observed on Dec. 23. Flares and dips are shown in colours as in Fig. 1. (See electronic version for colour figure.)

of width, $W_{1}=\left(1.0_{-0.5}^{+0.7}\right) \times 10^{-2}, W_{2}=0.19_{-0.05}^{+0.08}$, and $W_{3}=$ $4.0_{-1.0}^{+1.5} \mathrm{~Hz}$, adequately fit the power spectrum $\left(\chi^{2}=37 / 67\right)$.

The upper limit on the pulsation amplitude is meaningful only if a putative pulsar is isolated or belongs to a wide binary system $\left(P_{\text {orb }} \gg T_{\text {obs }}\right)$. In dM10 we reported a possible $4.32 \pm 0.01 \mathrm{~h}$ optical photometric periodicity, possibly associated with the binary orbital period. The XMM-Newton OM $U$ band light curve indicates the presence of a longer period $6.4 \pm 0.2 \mathrm{~h}$ (see also Sect.4). If the binary period is as short as $4.3-6.4 \mathrm{~h}$, the secondary star mass would be in the range 0.3-0.7 $M_{\odot}$ (Smith \& Dhillon 1998). We therefore need to consider shorter integration times in order to limit the leakage of power in close frequency bins due to the Doppler shift affecting the signal frequency. The optimal integration time to search for a signal coming from a binary system, without making any correction of the photon arrival times for the unknown orbital motion, was evaluated by Johnston \& Kulkarni (1991). Adopting as a lower limit the $4.3 \mathrm{~h}$ period and using the corresponding orbital parameters, one obtains

$T_{\text {best }} \simeq 247\left(\frac{v}{300 \mathrm{~Hz}}\right)^{-1 / 2}\left(\frac{P_{\text {orb }}}{4.3 \mathrm{~h}}\right)^{2 / 3}\left(\frac{M_{2}}{0.3 M_{\odot}}\right)^{-2 / 5}\left(\frac{\sin i}{\sin 45^{\circ}}\right)^{-1 / 2} \mathrm{~s}$.

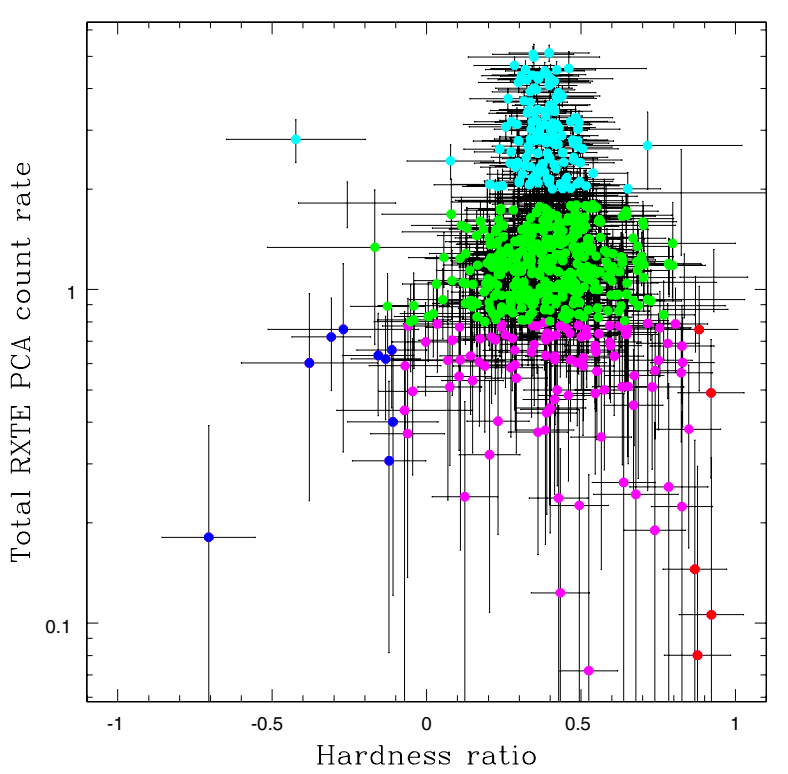

Fig. 4. HID between the $\mathrm{HR}_{\mathrm{RXTE}} 2-4 \mathrm{keV}$ and $4-9 \mathrm{keV}$ bands as defined in the text. Colours are used as in Fig. 2. (See electronic version for colour figure.) 


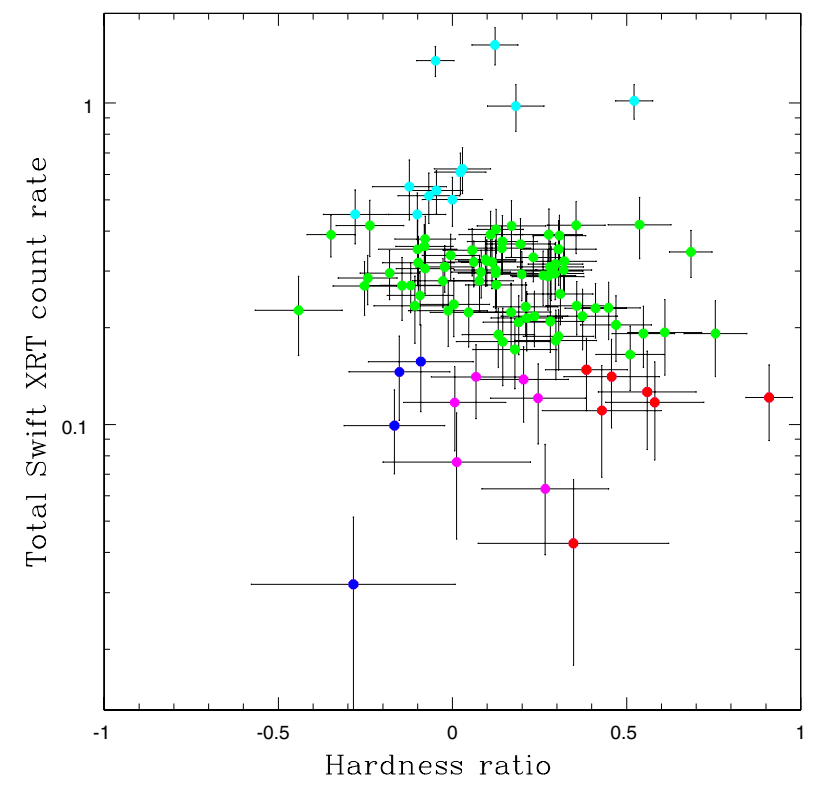

Fig. 5. HID between the $0.3-1.5 \mathrm{keV}$ and $1.5-10 \mathrm{keV}$ Swift/XRT bands. Colours are used as in previous figures. (See electronic version for colour figure.)

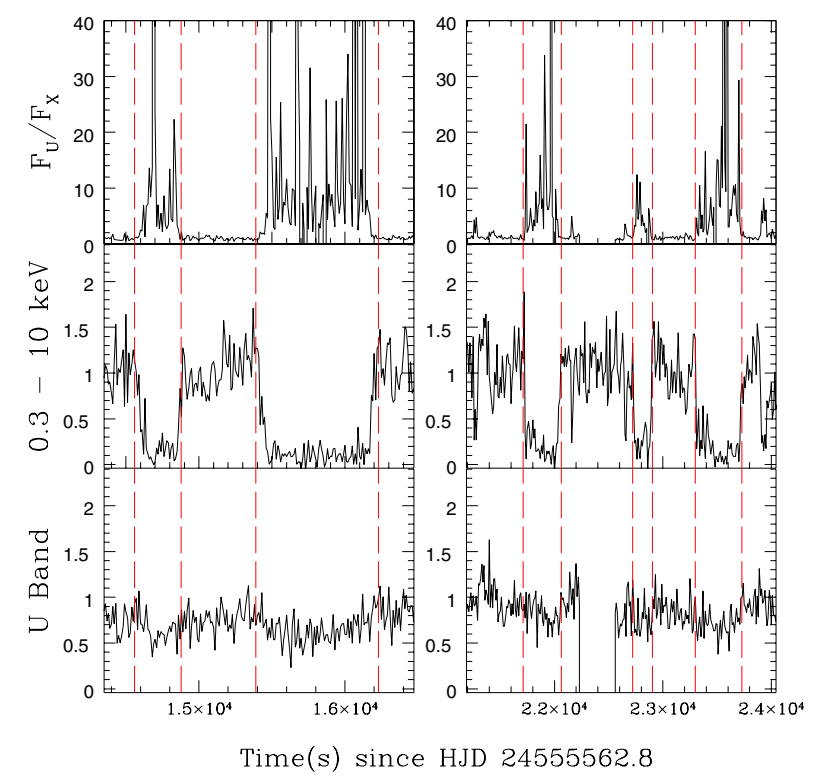

Fig. 6. Enlargement of the normalized light curves in 2011 showing the simultaneous occurrence of quiescent dips in the $U$ band (bottom panel) and X-rays (middle panel) $(0.3-10 \mathrm{keV})$ with a binning time of $10 \mathrm{~s}$, together with the ratio of the $U$ and X-ray band count rates (top). Vertical dashed (red) lines mark the dip start and end times.

Assuming that a pulsar in this LMXB system has a frequency in the range displayed by the known accreting pulsars with such low mass companions (100-600 Hz), one obtains optimal integration times spanning $150-395 \mathrm{~s}$. We produced several power spectra over time intervals of length 124 and $248 \mathrm{~s}^{1}$. No significant detection within a $3 \sigma$ confidence level was obtained. However, this is not surprising however, because at the low count

\footnotetext{
1 The lengths of the time intervals 124 s, 248 s, and 1983.7 s were chosen in order to give a number of bins equal to an integer power of $2^{19}$, $2^{20}$, and $2^{23}$, respectively. This greatly decreases the CPU time needed to perform fast Fourier transforms (FFT).
}
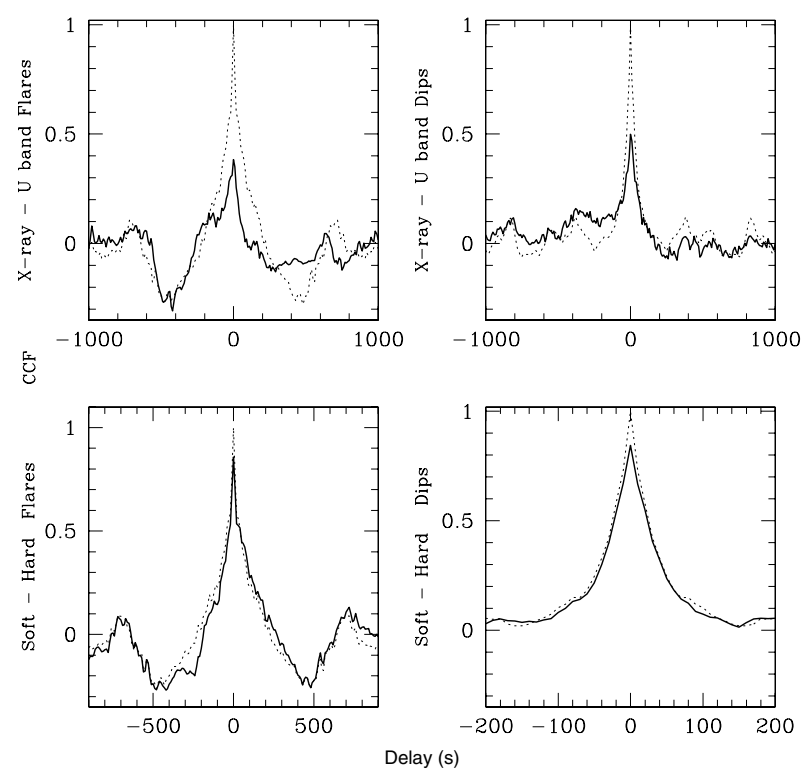

Fig. 7. CCFs (solid lines) during flares (left) and dips (right). The CCF between the hard and the soft X-ray bands, taking as reference the soft range (ACF), are shown in the bottom panels. The CCFs between the total X-ray and $U$ bands, taking as reference the X-ray band (ACF), are shown in the top panels. The ACF of each reference band is plotted with a dotted line.

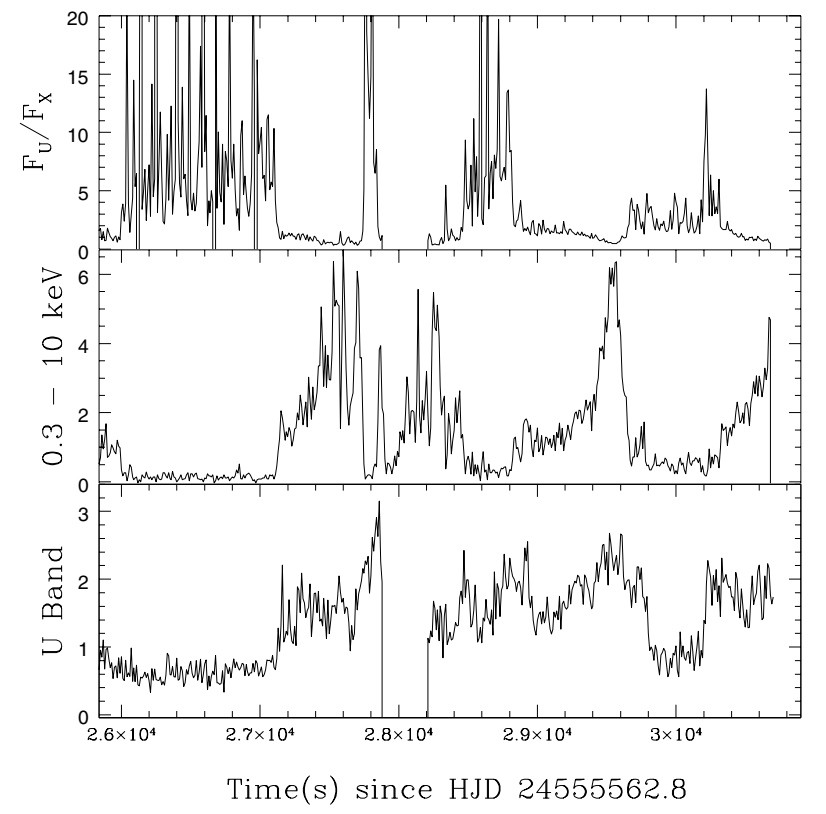

Fig. 8. Enlargement of the normalized light curves showing the flaring activity in the $U$ band (bottom panel) and X-rays (middle panel) $(0.3-10 \mathrm{keV})$ with a binning time of $10 \mathrm{~s}$, together with the ratio of the $U$ and X-ray band (top).

rates of this source, the number of counts in every interval is so low that only extremely loose $3 \sigma$ upper limits on the pulse amplitude of the order of $A_{\mathrm{UL}} \sim 0.5$ could be obtained.

In order to draw more stringent constraints on the presence of a coherent signal in the dataset, we applied quadratic coherence recovery techniques (QCRT; Wood et al. 1991; Vaughan et al. 1994). Such techniques rely on the correction of the times of arrival of the X-ray photons by using a quadratic time transformation, under the assumption that the sinusoidal Doppler 


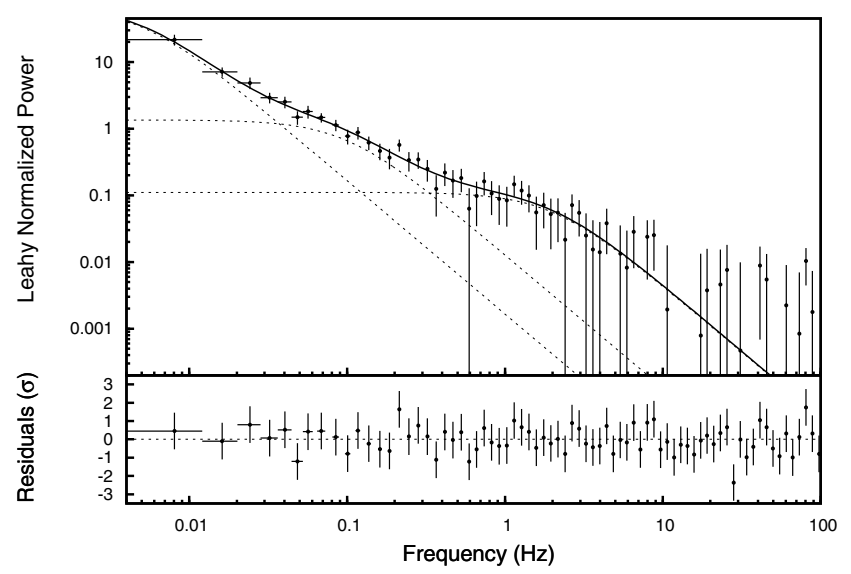

Fig. 9. Top panel: power spectrum obtained by averaging 242 intervals, each $\simeq 124$ s long, and logarithmically re-binning the spectrum with a factor of 1.1. The white noise level of 1.9880(4), evaluated by modelling the spectrum between 0.5 and $2 \mathrm{kHz}$ with a constant, is subtracted from each power estimate. Bottom panel: residuals in units of $\sigma$ of the power spectrum with respect to the best-fitting model composed of three lorentzians centred at zero frequency.

modulation of the arrival times introduced by the orbital motion is well approximated by a parabola over the considered integration time. The optimal integration time for the detection of a signal with QCRT was derived by Johnston \& Kulkarni (1991) as

$T_{\text {best }}^{\text {acc }} \simeq 1361\left(\frac{v}{300 \mathrm{~Hz}}\right)^{-1 / 3}\left(\frac{P_{\text {orb }}}{4.3 \mathrm{~h}}\right)^{7 / 9}\left(\frac{M_{2}}{0.3 M_{\odot}}\right)^{-4 / 15}\left(\frac{\sin i}{\sin 45^{\circ}}\right)^{-1 / 3} \mathrm{~s}$.

We therefore split the data in $N_{\text {intv }}=15$ intervals composed of $2^{23}$ bins, each of length equal to $t_{\text {bin }}$ as before, and performed FFT on the time series corrected with the relation $t^{\prime}=\alpha t^{2}$, where $\alpha=-1 / 2(a \sin i / c)(2 \pi v)\left(2 \pi / P_{\text {orb }}\right)^{2} \sin \phi_{0}, a \sin i / c$ is the projected semi-major axis of the primary star orbit and $\phi_{0}$ is the orbital phase at the beginning of the time series. The maximum and minimum values of $\alpha$, as well as the value $\delta \alpha$ by which $\alpha$ is incremented in each of the corrections, have been determined by Vaughan et al. (1994) as $\alpha_{\max }=-\alpha_{\min }=$ $8.80 \times 10^{-8}(q / 0.29)\left(P_{\text {orb }} / 4.3 \mathrm{~h}\right)^{-4 / 3}\left(M_{\text {tot }} / 1.7 M_{\odot}\right)^{1 / 3} \mathrm{~s}^{-1}$, and $\delta \alpha=1 /\left(2 v_{\mathrm{Ny}} T^{2}\right)$. By considering the range of values for the parameters of the binary system and the reported uncertainties, a range of masses between 1.4 and $2.0 M_{\odot}$ for the mass of a putative NS in this system, $v_{\mathrm{Ny}} \simeq 2114.35 \mathrm{~Hz}$ and $T_{\mathrm{FFT}} \simeq 1983.7 \mathrm{~s}$, we set $\alpha_{\max }=-\alpha_{\min }=1 \times 10^{-7} \mathrm{~s}^{-1}$ and $\delta \alpha=6 \times 10^{-11} \mathrm{~s}^{-1}$ so that $N_{\alpha}=3334$ corrections were performed on each of the $N_{\text {intv }}=15$ time intervals. The detection threshold is therefore $P_{\text {det }}=62.58$ in the frequency range, where the noise is compatible with being distributed as a chi-squared with two d.o.f. (see Vaughan et al. 1994). No signal above this level was found. The upper limits at $3 \sigma$ confidence level set on the pulse amplitude in each of the intervals are plotted in Fig. 10, together with the light curve in the $0.5-10 \mathrm{keV}$ band. The values we find as limits on the pulse amplitude $(\sim 0.15-0.25)$ are larger than those usually measured from pulsars in LMXB systems.

\subsection{Persistent $X$-ray and gamma-ray emissions}

The X-ray history of XSS J1227 collected over $7 \mathrm{yr}$ shows that it is a persistent and rather stable X-ray source, with flares and

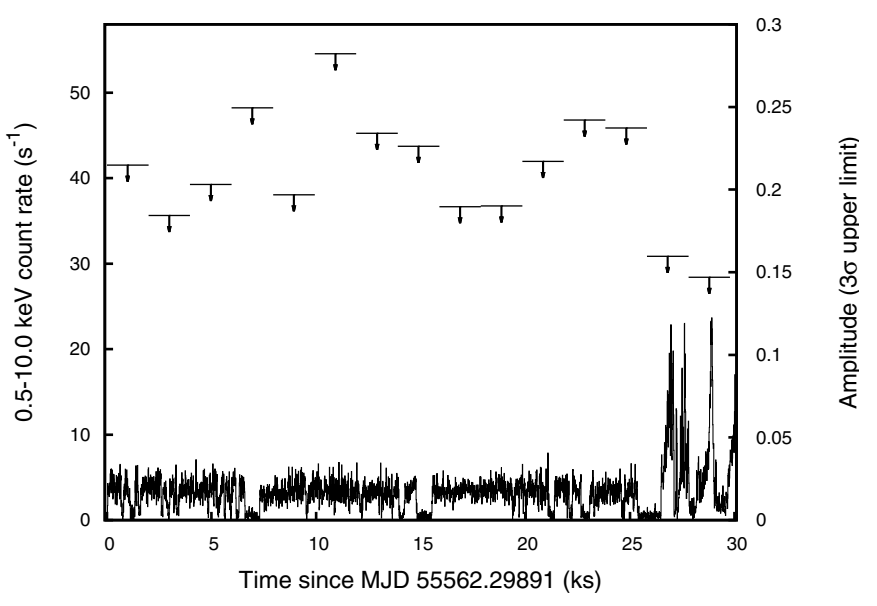

Fig. 10. Right scale: upper limits at a $3 \sigma$ confidence level on the pulse amplitude during the EPIC-pn observation of XSS J1227, as obtained by performing a QCRT over $1983.7 \mathrm{~s}$ long intervals (see text). Left scale: the net $0.5-10 \mathrm{keV}$ light curve recorded by the EPIC-pn is also over-plotted.

dips being peculiar and permanent characteristics. The associated Fermi-LAT source 2FGL J1227 is reported in both 1st and 2nd catalogues to be non-variable on a monthly timescale over the first two years of Fermi operations (see Nolan et al. 2012) and also on timescale $>4 \mathrm{~d}$ in the $0.1-300 \mathrm{GeV}$ range over the first 25.5 months of Fermi-LAT observations (Hill et al. 2011).

We then extended the gamma-ray coverage in the 0.1-300 GeV range up to Apr. 18, 2012 and show the whole light curve (Fig. 11) with the same $4 \mathrm{~d}$ binning as Hill et al. (2011). The average photon flux is $\sim 1 \times 10^{-7}$ photons $\mathrm{cm}^{-2} \mathrm{~s}^{-1}$, slightly lower but consistent with that obtained by Hill et al. (2011). Differences are due to the different photon selection criteria and IRF adopted at the two epochs of data analysis ${ }^{2}$ We have also overlaid the X-ray pointings since 2008, from which it is possible to conclude that both X-ray and gamma-ray emissions are detected simultaneously. Since a shorter time bin size is precluded by the low photon flux, it is not possible to infer whether the gamma rays are emitted steadily or bursts or flares.

\section{The spectral characteristics}

In $\mathrm{dM} 10$ we reported the analysis of the average X-ray spectrum in the $0.3-100 \mathrm{keV}$ range using XMM-Newton, RXTE, and INTEGRAL. The spectrum is featureless and well fitted with an absorbed power-law with $\Gamma=1.7 \pm 0.02$. Similar results were found by Saitou et al. (2009). The spectral shape was also found to be invariant to flux variations, e.g. during quiescence, quiescent dips, and flares, but not during the hard postflare dips, where a partial covering absorber better described the spectrum (dM10). We have further checked this behaviour using the 2011 XMM-Newton EPIC-pn data, fitting them with an absorbed power-law and obtained similar results: $\Gamma=1.64 \pm 0.01$ (quiescence), $\Gamma=1.65 \pm 0.03$ (flares), $\Gamma=1.71 \pm 0.04$ (quiescent dips), and $\Gamma=0.74 \pm 0.08$ (post-flare dips). The column density of the absorber is within errors the same in all fits and similar to that found in the 2009 XMM-Newton data,

\footnotetext{
2 Details of reccomendation for data selection at the Fermi Science Support Centre: http://fermi.gsfc.nasa. gov/ssc/data/analysis/documentation/Cicerone/ Cicerone_Data_Exploration/Data_preparation.html
} 


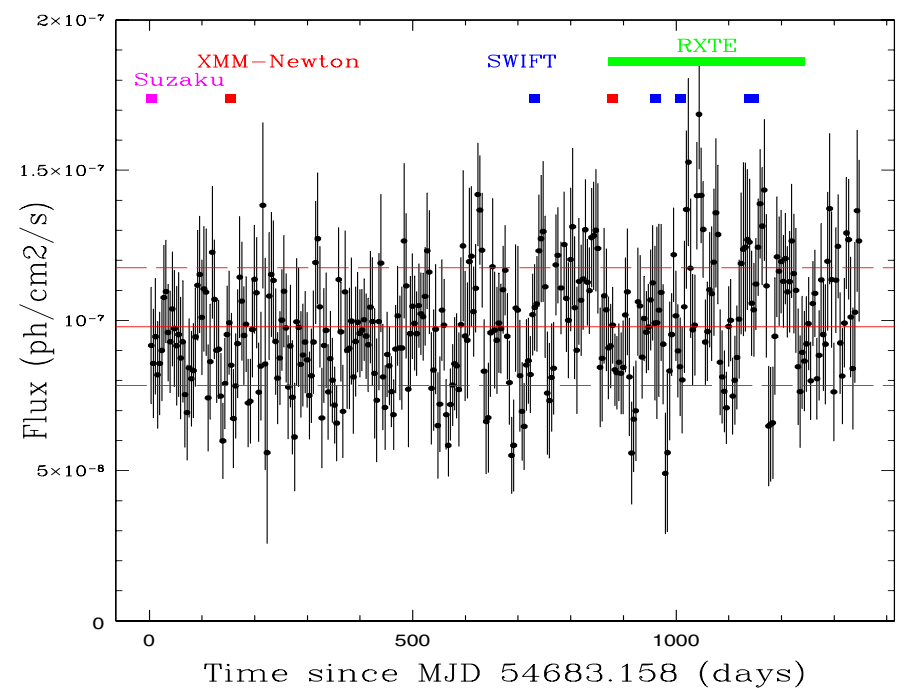

Fig. 11. Fermi-LAT light curve in the range $0.1-300 \mathrm{GeV}$ with a $4 \mathrm{~d}$ binning time from Aug. 2008 to Apr. 2012 extracted in a $1^{\circ}$ aperture. The mean flux is reported together with the $1 \sigma$ standard deviation. The background is not subtracted. The X-ray coverages by Suzaku (magenta), RXTE (green), XMM-Newton (red), and Swift (blue) are also reported. (See electronic version for colour figure.)

$N_{\mathrm{H}}=0.75 \pm 0.04 \times 10^{21} \mathrm{~cm}^{-2}$, and consistent with the galactic medium in the direction of the source. The fit to the post-flare dip spectrum improves when adding a partial covering absorber. We find $\chi^{2} /$ d.o.f. $=33 / 25$ vs. $\chi^{2} /$ d.o.f. $=43 / 27$ with and without this component. This component is found to be significant at the $2.1 \sigma$ confidence level. The column density and partial covering fraction of the absorber are $3.85_{-1.4}^{+2.5} \times 10^{21} \mathrm{~cm}^{-2}$ and $0.61 \pm 0.2$, respectively. Also, fits to the RXTE quiescent and flare spectra give similar power-law indexes, further corroborating the unvariancy of the spectrum during active and persistent level. The spectrum of the quiescent dips might indicate a softening, but it does not require an additional soft component. The $2-10 \mathrm{keV}$ fluxes as derived for the 2011 XMM-Newton data are $0.26 \pm 0.04$, $3.12 \pm 0.1,0.98 \pm 0.05$, and $1.4 \pm 0.1 \times 10^{-11} \mathrm{erg} \mathrm{cm}^{-2} \mathrm{~s}^{-1}$ during the quiescent dips, the flares, the post-flare dips, and quiescence, respectively. These are consistent with those found in 2009 (dM10).

To construct the broad-band SED we implemented the X-ray unabsorbed spectrum obtained in dM10 using the XMM-Newton, RXTE, and INTEGRAL data. This is reported in Fig. 12 together with the best-fit power-law to the quiescent X-ray average spectrum. We also overlaid the extinction-corrected XMM-Newton OM UV and $U$ band average fluxes (dM10), the extinctioncorrected optical spectrum by Masetti et al. (2006), and the 2MASS nIR fluxes (see dM10 for details on extinction). The UV/optical and nIR data are consistent with each other, but are off by more than one order of magnitude from that extrapolating the X-ray power-law. The simultaneity of the UV and $U$ band data with the X-ray data then implies a different component contributing to the UV/optical/nIR flux. We therefore analysed the UV/optical/nIR SED and found that it is best fitted by a composite function consisting of two blackbodies at $T_{\mathrm{h}}=$ $12800 \pm 600 \mathrm{~K}$ and $T_{\mathrm{c}}=4600 \pm 250 \mathrm{~K}$ (uncertainty at $1 \sigma$ c.l.) $\left(\chi^{2} /\right.$ d.o.f. $\left.=11 / 20\right)$. In Fig. 13 we report the UV/optical $/ \mathrm{nIR}$ SED, where the optical spectrum from (Masetti et al. 2006) was rebinned after removal of emission lines together with the twocomponent blackbody function. The latter is also reported in
XSSJ12270-4859

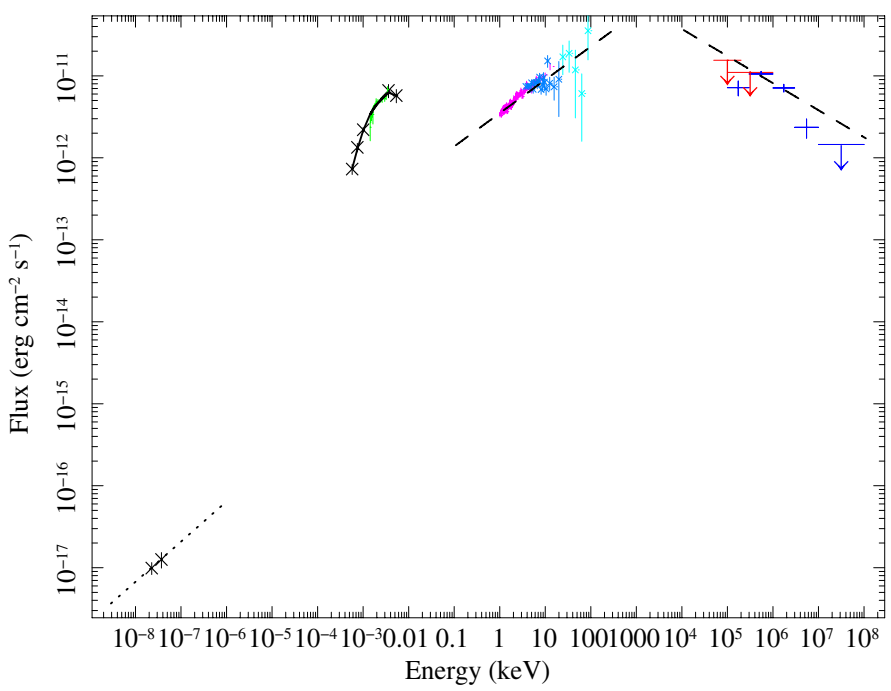

Fig. 12. Combined unabsorbed SED using the gamma-ray fluxes from Fermi-LAT (blue) and AGILE/GRID (red), the XMM-Newton (magenta), RXTE (light blue), and INTEGRAL (cyan) average X-ray spectrum, the XMM-Newton OM UV and $U$ band (black stars) fluxes from dM10, the optical spectrum from Masetti et al. (2006) (green), the 2MASS nIR measures and the ATCA radio measures from Hill et al. (2011) (black stars). Overlaid are the simple power-law forms derived from the X-ray and gamma-ray spectra and from the radio-flux ratios as well as the best-fit composite blackbody model to the UV/optical/nIR fluxes. (See electronic version for colour figure.)

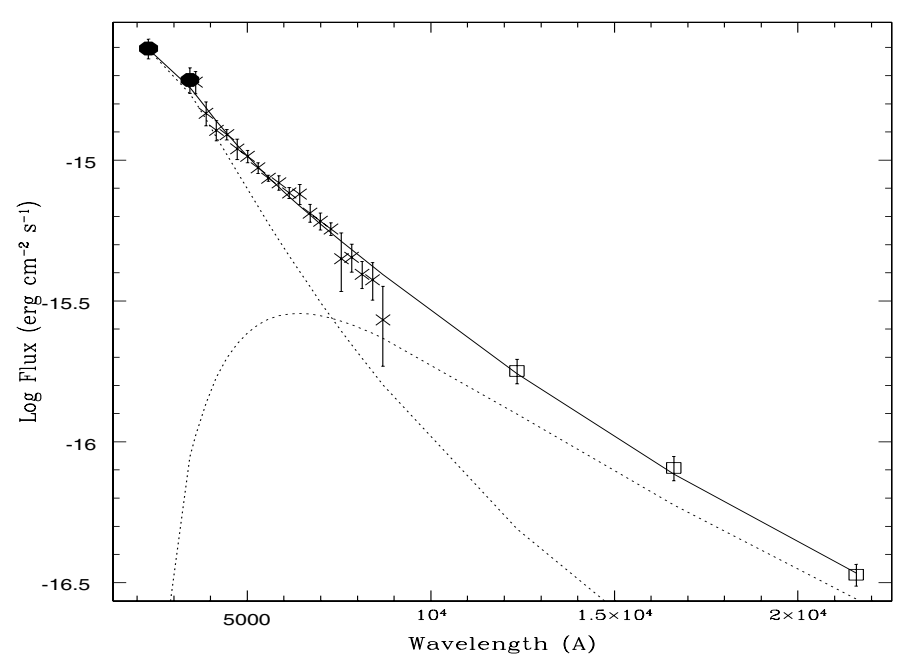

Fig. 13. Unabsorbed UV to nIR SED, obtained combining the $X M M-N e w t o n$ OM UVM2 and $U$ band fluxes (filled circles), the optical spectrum from Masetti et al. (2006) (crosses), and the 2MASS fluxes (empty squares) together with the best-fit composite (solid line) function with two blackbodies with temperatures of $12800 \mathrm{~K}$ and $4600 \mathrm{~K}$ (dotted lines).

Fig. 12. The projected areas of the hot component is $5 \%$ of the cool one. The low-temperature blackbody could be compatible with that of a late-type star in the range $\mathrm{K} 2-\mathrm{K} 5 \mathrm{~V}$, which is of earlier type than what is estimated in $\mathrm{dM} 10$, assuming an orbital period of $4.3 \mathrm{~h}$. For a K2-K5 V star $(\log g=4.5)$, the projected area would imply a distance of $1.7 \pm 0.3 \mathrm{kpc}$. The $J$ and $K$ band fluxes of this component are $98 \%$ and $99 \%$ the 
total observed fluxes, respectively. Hence, it is reasonable to assume that the nIR flux is almost totally dominated by the donor star. We then also use the lowest $J$-band magnitude observed in dM10 photometry $(J=16.9)$, corrected for interstellar absorption $A_{J}=0.12 \mathrm{mag}$ (dM10), and the absolute magnitudes corresponding to a K2-K5 V star (Knigge 2006), thus obtaining a distance of $2.3-3.6 \mathrm{kpc}$. If the secondary star is indeed of $K$-spectral type, it would in turn indicate a binary period in the range 7-9h (Smith \& Dhillon 1998). These values and the $\sim 6 \mathrm{~h}$ variability detected in the near-UV might suggest a longer orbital period. The high-temperature component instead suggests a non-stellar contribution from an accretion disc or flow, because the optical spectrum is characterized by emission lines. The projected area of the hot emitting region is $\sim 7 \times 10^{20} d_{1 \mathrm{kpc}}^{2} \mathrm{~cm}^{2}$.

Assuming the association with the 2FGL source, we also constructed the gamma-ray portion of the spectrum using the AGILE/GRID upper limits and the Fermi-LAT fluxes as listed in the 2nd source catalogues (Fig. 12). The spectrum is also reported together with the best-fit 2FGL catalogue power-law with photon index 2.33. The high-energy portion of the gamma-ray spectrum might suggest a decay that Hill et al. (2011) fitted with an exponential cut-off at $\sim 4.1 \mathrm{GeV}$, found to be significant at $4 \sigma$. The logParabola used in the 2FGL catalogue could also support this feature. The AGILE/GRID upper limits are roughly compatible with the Fermi-LAT measures. The gamma-ray SED does not support a peak in the $1-100 \mathrm{MeV}$ range, as suggested in $\mathrm{dM} 10$. Therefore there are no strong evidences that the X-ray and gamma-ray fluxes are linked.

We furthermore include the dual band ATCA fluxes at 5.5 GHz and $9 \mathrm{GHz}$ from Hill et al. (2011), acquired in 2009, of the radio counterpart to XSS J1227. Although not contemporaneous, the X-ray and gamma-ray histories allow us to adopt the radio measures for the broad-band SED. The flux-density ratio at these frequencies, assuming a power-law distribution $S_{v} \propto v^{\alpha}$, gives $\alpha=-0.5$ (Hill et al. 2011). Taken at its face value, it is consistent with optically thin synchrotron emission. An extrapolation of this power-law to higher energies falls well below the $\mathrm{X}$-ray spectrum but roughly matches the soft gamma-ray flux at $\sim 100 \mathrm{MeV}$. However, we note that the large uncertainties in the spectral index derived by Hill et al. (2011) may also allow a flat radio spectrum.

\section{Discussion}

We have presented new X-ray data of XSS J1227 and new gamma-ray data of its positionally associated Fermi-LAT counterpart to infer the long-term temporal history and the possible nature of this intriguing source.

\subsection{A steady source: the persistent, flaring, and dipping states}

XSS J1227 is found at about the same flux level $\left(F_{2-10 \mathrm{keV}} \sim 1 \times\right.$ $10^{-11} \mathrm{erg} \mathrm{cm}^{-2} \mathrm{~s}^{-1}$ ) over $7 \mathrm{yr}$ of observations, displaying peculiar short-term behaviour with flares and dips in all observations. Flares are simultaneously detected in both X-rays and UV band on timescales of $\sim 600-850 \mathrm{~s}$. All X-ray flares occur in the form of flare-dip pairs. In dM10 we drew comparison with type-II bursts sources, although with different flare energetics and duration. Flares appear to occur on a timescale of $\gtrsim 9-11 \mathrm{~h}$ and are grouped in multiple shorter events with a total duration of $\gtrsim 1 \mathrm{~h}$. Flares were also reported to occur in the nIR band by Saitou et al. (2011). The large variability $(\sim 0.4 \mathrm{mag})$ detected in our previous $J$-band data (dM10) was not recognized to be in the form of flares due to the poor statistics. The optical photometry by Pretorius (2009) and dM10 also shows large-amplitude short term variations that could be affected by flares (dM10).

While X-ray flares are characterized by unvariant spectral shape with respect to the quiescent persistent level, the post-flare dips involve absorbing material that is dense $\left(N_{\mathrm{H}}=\right.$ $\left.4 \times 10^{21} \mathrm{~cm}^{-2}\right)$, partially $(\sim 60 \%)$ covering the X-ray source. The simultaneous occurrence of flares at lower energies in the nearUV corroborates the interpretation given in dM10, where flaredip pair events are due to accretion events onto the compact object followed by a slow refilling of the missing regions of an accretion disc. The emptied region should be extended because it is detected at UV and near-UV energies.

On the other hand, the ordinary dips observed during quiescence in X-rays and near-UV appear to occur randomly. Here we note that optical ground-based photometry does not reveal such sharp dips (Pretorius 2009, dM10), indicating that dips originate close to the compact star. The sharpness of their profiles, the different lengths (from $\sim 15 \mathrm{~s}$ up to $\sim 1100 \mathrm{~s}$ ), the timescales of decays/rises and the invariance (or possibly slight softening) of the X-ray spectrum might point to occultation due to discrete material. If this matter is located at the disc edge, the dip ingresses/egresses might be used to estimate the size of the eclipsed X-ray emitting region, similar to LMXB dippers. However, LMXB dippers are known to show intensified dipping activity at specific orbital phases with hardening of the spectrum due to absorbing material located close to the disc bulge (Boirin et al. 2005; Iaria et al. 2007; Diaz Trigo et al. 2009). The frequent and apparently non-periodic occurrence of deep dips in XSS J1227 could be related to opaque material distributed randomly above the disc. Although caution should be taken, we follow Church \& Balucinska-Church (2004) for LMXB dippers, using the longer dips where the X-ray count rate drops to zero. The range of ingress/egress times is $\sim 40-90 \mathrm{~s}$. We assume an accretion disc radius $R_{\text {disc }} \sim 0.90 R_{\text {Lobe, } 1 \text {. Given the uncertainty }}$ in the orbital period, we use the wide range $\sim 4-9 \mathrm{~h}$ and adopt a mass ratio $q=0.8$. With these values, the mass of the companion star would be in the range $0.4-1 M_{\odot}$ (Smith \& Dhillon 1998) and the disc radius would result $\sim 4-5 \times 10^{10} \mathrm{~cm}$. If the material is located at this distance and the angular size of the material is larger than the X-ray emitting accretion disc corona (ADC, see Eq. (1) of Church \& Balucinska-Church 2004), the time of ingress is related to the radius of the emitter. In this case, we obtain $R_{\mathrm{ADC}} \sim 2-8 \times 10^{8} \mathrm{~cm}$. This is about one order of magnitude smaller than those determined for LMXB dippers (Church \& Balucinska-Church 2004). The size of the ADC scales with the X-ray luminosity (Church \& Balucinska-Church 2004) would imply $L_{X} \sim 0.5-1.0 \times 10^{36} \mathrm{erg} \mathrm{s}^{-1}$. This is about two orders of magnitude larger than that derived for a distance of $1 \mathrm{kpc}(\mathrm{dM} 10)$. Even at distances of $2.4-3.6 \mathrm{kpc}$, the X-ray (bolometric) luminosity is lower $\left(3-8 \times 10^{34} \mathrm{erg} \mathrm{s}^{-1}\right)$. Therefore, either the disc corona is limited in extension or the occulting material is not located at the adopted disc rim.

In addition, the dips are observed in the UV, implying that the hot $13 \mathrm{kK}$ component that dominates the UV light also suffers occultation. This component has an emitting area of $\pi\left(0.2 R_{\odot}\right)^{2} d_{1 \mathrm{kpc}}^{2}$. This is smaller than the adopted disc radius but larger than those determined for similar hot components in LMXBs and attributed to the bright disc hot spots or a region closer to the centre (Froning et al. 2011; Hynes \& Robinson 2012). The UV/optical emitting region is larger than that estimated for the X-ray emitter. This can explain why the UV flux 
deficiency is lower $(\sim 40 \%)$ than that in the X-ray band during the dips.

Furthermore, the fact that the ratio of bolometric fluxes between the X-ray and UV components is $\sim 6$ and the X-ray peak intensity at flares is about twice that in the UV indicates strongly that the UV light originates from X-ray reprocessing in a larger region surrounding the $\mathrm{X}$-ray source.

In summary, the long-term X-ray history of XSS J1227 over $7 \mathrm{yr}$ and the gamma-ray light curve of the associated source 2FGL J1227 over $4.7 \mathrm{yr}$ indicate that this peculiar binary is a persistent source in both energy ranges and that it always displays the same X-ray variability characteristics. We cannot, however, exclude the possibility that the gamma rays are also emitted in the form of flares with shorter timescales than $4 \mathrm{~d}$.

\subsection{An LMXB hosting an MSP?}

Analogies to the MSP binary PSR J1023+0038 (Tam et al. 2010) were suggested by Hill et al. (2011) because of similarities of the optical spectra (both sources were formerly proposed as CVs), the existence of radio and Fermi-LAT counterparts and the short, putative $\sim 4 \mathrm{~h}$ orbital period, and large optical flickering. PSR J1023+0038 was proposed to be active in gamma rays when accretion switches off and evidence of accretion stopping at least once comes from an optical, although non-simultaneous, spectrum (see Tam et al. 2010 and reference therein). The possibility that accretion switches off in XSS J1227 was left open in Hill et al. (2011), but the present study strongly indicates that the source has always been in an accretion state. This is further confirmed by recently acquired optical spectroscopy (de Martino et al., in prep.).

We draw a further comparison with MSP binaries detected by Fermi. The gamma-ray spectra of PSR J1023+0038 and 2FGL J1227 are rather similar, with a steep power-law in$\operatorname{dex} \Gamma 2.5$ (in PSR J1023+0038) and $\Gamma=2.3$ in 2FGL J1227. Although not strongly constrained, a power-law exponential cutoff model for PSR J1023+0038 gave $\Gamma=1.9$ and $E_{\text {cutoff }}=$ $0.7 \mathrm{GeV}$ (Tam et al. 2010) as compared with $\Gamma=2.2$ and $E_{\text {cutoff }}=4.1 \mathrm{GeV}$ inferred for 2FGL J1227 by Hill et al. (2011). Ransom et al. (2011) fitted the gamma-ray spectra of PSR J06143329, PSR J1231-1411 and PSR J2214+3000, with a power-law exponential cutoff, obtaining $E_{\text {cutoff }}$ in the range $2.5-4.5 \mathrm{GeV}$ and $\Gamma \sim 1$.4. Kerr et al. (2012) find for PSR J0101-6422 $E_{\text {cutoff }}=$ $1.9 \mathrm{GeV}$ and $\Gamma=0.9$. Also for the very recently discovered black-widow MSP PSR J1816+4510, Kaplan et al. (2012) found $\Gamma=2$ and $E_{\text {cutoff }} \sim 7.5 \mathrm{GeV}$. Therefore, based on the gamma-ray spectrum, 2FGL J1227 is not much different from the variety of MSP binaries recently discovered by Fermi.

The X-ray $(0.2-100 \mathrm{keV})$ to gamma-ray $(0.1-100 \mathrm{GeV}) \mathrm{lu}-$ minosity ratio of our source is $\sim 0.8$, whilst most of the MSP in LMXBs have lower ratios by $2-3$ orders of magnitudes (Bogdanov et al. 2006, 2011; Ransom et al. 2011). Our exploration of the overall SED also indicates that the X-ray flux, dominated by a non-thermal component, is higher than that expected from rotation-power MSP emission. In the latter, the thermal flux from the NS atmosphere either dominates (Ransom et al. 2011) or is detectable, as in the case of PSR J1023+0038 with a thermal fraction $\sim 3-6 \%$ (Bogdanov et al. 2011; see also Takata et al. 2012). The non-thermal X-ray emission, if originating from an intrabinary shock, such as in the blackwidow MSPs, would be the extension of the gamma-ray flux at these energies (Takata et al. 2012), and this is not the case for XSS J1227. Intrabinary shock as source of non-thermal emission in the quiescent MSP binary PSR J1023+0038 was proposed by Bogdanov et al. (2011).

Our search for X-ray msec pulses in XSS J1227 only provided upper limits $\sim 15 \%$ to pulse amplitudes. In accreting MSPs, pulse amplitudes in excess of $10 \%$ are rarely observed, but fractional amplitudes can range between $0.43 \%$ and $\sim 30-40 \%$ (see review by Patruno \& Watts 2012). Here we note that in PSR J1023+0038 the X-ray pulse amplitude was found to be $11 \pm 2 \%$ (Archibald et al. 2010). Therefore, we cannot exclude that XSS J1227 harbours a fast-spinning pulsar. However, in non-accreting MPS the X-ray pulses would arise from the NS atmosphere or hot spots that we have not detected in the X-ray spectrum of XSS J1227. A non-thermal component arising from intrabinary shock would only produce an X-ray orbital modulation, as is the case of PSR J1023+0038, which shows large amplitude orbital variability (Bogdanov et al. 2011). While in the 2009 XMM-Newton data, a possible weak X-ray variability could be present, this is not detected in the higher quality EPICpn data in 2011. Hence, all this points to an accretion-driven $\mathrm{X}$-ray emission.

Accretion acting in this source is further corroborated by the UV/optical/nIR portion of the SED, which reveals two spectral components: a hot one at $\sim 13 \mathrm{kK}$ and a cool one at $4.6 \mathrm{kK}$. A hot, but single, spectral component was also recently identified in the UV/optical SED of the MSP binary PSR J1816+4510 (Kaplan et al. 2012), making it the black-widow binary pulsar hosting the hottest low-mass companion $(\gtrsim 10 \mathrm{kK})$. We however note that no nIR data are available to date for PSR J1816+4510. In XSS J1227, the cool component is instead revealed from the nIR 2MASS measures and its temperature suggests the contribution from a late-type K2-K5 companion, earlier than that estimated in $\mathrm{dM} 10$. Hence, the UV/optical component can be reasonably ascribed to accretion. The temperature is, however, higher than that of accretion discs (see Hynes \& Robinson 2012) and the size of the emitting region could suggest that it originates closer to the compact object. The X-ray and UV bolometric luminosity ratio is $\sim 6$, compatible with an $\mathrm{X}$-ray heated region. We note that this ratio has been proposed to be a discriminant between NS and $\mathrm{BH}$ binaries, with NS having X-ray-to-UV flux ratios about ten times higher than BH systems (Hynes \& Robinson 2012).

If the radio spectral power-law index is truly $\alpha=-0.5$, then the extrapolation to the gamma-ray regime roughly matches the observed flux at $\sim 100 \mathrm{MeV}$. However, searches for fast pulses in the radio counterpart of XSS J1227 failed (Hill et al. 2011). Therefore, while the flux ratio could be consistent with nonthermal emission, the lack of detection of pulses does not favour a rotation-powered radio pulsar.

\subsection{An LMXB powering a jet?}

The hard X-ray spectrum and the low-luminosity of XSS J1227 indicate an LMXB in a deep faint hard state. From the detection of quasi-simultaneous X-ray and nIR flares and the low X-ray luminosity, Saitou et al. (2011) argued that XSS J1227 could be a microquasar with a synchrotron jet in a prolonged low-luminosity state at $10^{-4} L_{\text {Edd }}$ for a stellar mass BH or NS at $1 \mathrm{kpc}$. As a consequence of synchrotron cooling in an optically thin medium, the energy break expected in these binaries is at $\sim 500 \mathrm{keV}$ (Saitou et al. 2011), much lower than that detected at $\sim 4 \mathrm{GeV}$ in 2FGL J1227.

High-energy gamma rays were detected in galactic binaries hosting high-mass early-type stars, such as LS 5039, LSI +61³03 (Abdo et al. 2009a,b; Hadasch et al. 2012), 1FGL J1018.6+5856 (Ackermann et al. 2012), and Cyg X-3 
during flares (Tavani et al. 2009b; Abdo et al. 2009c). The latter is the only gamma-ray source with a BH candidate while the others are proposed to possibly harbour NSs. The gamma-ray spectrum of $\mathrm{LSI}+61^{\circ} 303$ is compatible with a power-law exponential cut-off at $\sim 4 \mathrm{GeV}$ (Abdo et al. 2009a; Hadasch et al. 2012), while LS 5039 shows a power-law exponential cut-off at $\sim 2 \mathrm{GeV}$ (Abdo et al. 2009b; Hadasch et al. 2012). The nature of GeV emission from these binaries is still unclear and controversial (Hadasch et al. 2012; Papitto et al. 2012; Torres et al. 2012). They could be powered either by magnetospheric emission, as seen in many gamma-ray detected pulsars, or by electrons in the jets accelerated up to $\mathrm{TeV}$ energies (leptonic model; Hadasch et al. 2012). For Cyg X-3, the Fermi flux is compatible with the extrapolation of the X-ray tail to $100 \mathrm{MeV}$ and hence is believed to be the high-energy tail of the soft gamma-ray spectral component. Also, in this case the origin of gamma-ray photons is unclear and is proposed to be leptonic (Abdo et al. 2009c). Since no LMXB with a radio jet has been detected in the gamma rays so far, it is difficult to predict the origin of high-energy gamma-ray emission.

We alternatively explored the radio-to-X-ray emission from LMXBs hosting NS and $\mathrm{BH}$ in the hard faint quiescent states. The persistent radio emission from $\mathrm{BH}$ binaries during these states is generally consistent with a flat or slightly inverted spectrum that, in analogy with compact extragalactic radio sources, is attributed to the superimposition of a number of peaked synchrotron spectra generated in a jet (Gallo 2010). Atoll NS binaries in low-hard states (island states) have also been detected at radio frequencies, though most are upper limits with a handful of systems detected simultaneously with the X-rays (Migliari \& Fender 2006). Usually, dual-band radio spectra are not well constrained and could be consistent with flat $(\alpha \simeq 0)$ or optically thin $\alpha \sim-0.6$ slopes, such as Aql X-1 or $4 \mathrm{U} 1728-34$ (Migliari et al. 2003; Migliari \& Fender 2006). In NS binaries, jets can nevertheless be powered during these low states but are about one order of magnitude less powerful than BH jets. The radio spectrum of XSS J1227 is not well constrained, $\alpha=0.5 \pm 0.6$ (Hill et al. 2011), leaving the possibility of a similar origin as those in $\mathrm{BH}$ or NS binaries.

The $\mathrm{BH}$ binaries spend most of their time in such states, which could be compared to the prolonged low-state of XSS J1227. The X-ray emission from these binaries originates in an optically thick accretion disc with a hard component, which could be an ADAF-like (advection dominated accretion flow; Narayan \& Yi 1994) or from a jet. The jets are coupled with the accretion flow, although the disc-jet connection is still unclear. The radio emission from the jet was found to be correlated with that in the X-rays during the hard state: $F_{\text {radio }} \propto F_{\mathrm{X}}^{b}$, with $b \sim 0.5-0.7$ (Gallo et al. 2003). A similar relation was found for atoll NS binaries in the island states but with a steeper correlation coefficient $\mathrm{b} \sim 1.4$ (Migliari \& Fender 2006). Hence, the X-ray emission in the $\mathrm{BH}$ systems is radiatively inefficient, with an approximate relation $L_{\mathrm{X}} \propto \dot{M}^{2}$, whilst the jet power in both classes scales linearly with the accretion rate. Keeping in mind the large uncertainty in the radio spectral slope, we have explored the possibility that XSS J1227 could host a compact jet following Coriat et al. (2011), where the radio/X-ray luminosities of $\mathrm{BH}$ binaries and NS LMXBs are compared. This is depicted in Fig. 14, adapted from Fig. 5 of Coriat et al. (2011). Here, the radio $(9 \mathrm{GHz})$ luminosity against the X-ray $3-9 \mathrm{keV}$ unabsorbed luminosity is shown for a sample of $\mathrm{BH}$ candidates in LMXBs (GX 339-4, H 1743-322 and V404 Cyg) during hard $\mathrm{X}$-ray states and for the two atoll NS binaries in the island state,

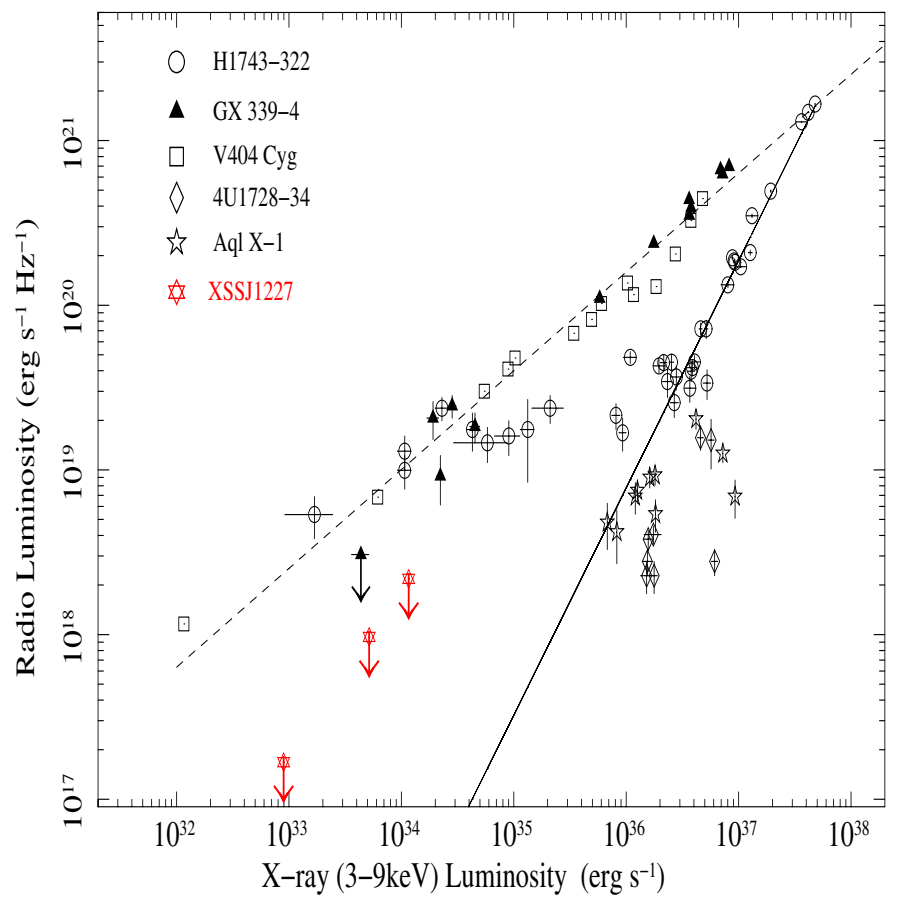

Fig. 14. Radio luminosity at $\sim 9 \mathrm{GHz}$ versus $3-9 \mathrm{keV}$ X-ray luminosity for the BH candidates GX 339-4, H 1743-322 and V404 Cyg during their hard X-ray states, together with the atoll-type NS binaries Aql X-1 and 4U 1728-34 during their island states (see footnote 2 for details on the data). The radio/X-ray luminosity correlation $F_{\text {radio }} \propto F_{\mathrm{X}}^{\mathrm{b}}$ with $b \sim 0.6$ for $\mathrm{BH}$ binaries and $b \sim 1.38$ for island state NS binaries is also reported. The positions of XSS J1227 at the three distances $1 \mathrm{kpc}, 2.4 \mathrm{kpc}$, and $3.6 \mathrm{kpc}$ are marked in red. Adapted from Coriat et al. (2011). (See electronic version for colour figure.)

Aql X-1 and $4 \mathrm{U} 1728-34^{3}$ In the radio vs. X-ray luminosity diagram, the $\mathrm{BH}$ candidate $\mathrm{H} 1743-322$ moves to lower X-ray luminosities, transiting the two correlations and joining the standard BH correlation at low luminosities (Coriat et al. 2011). This is the first $\mathrm{BH}$ candidate to follow both correlations depending on the X-ray luminosity.

We locate XSS J1227 in this diagram by using the ATCA $9 \mathrm{GHz}$ and $3-9 \mathrm{keV}$ fluxes adopting the three values for the distance, $1 \mathrm{kpc}, 2.4 \mathrm{kpc}$, and $3.6 \mathrm{kpc}$. For the sake of caution, these are marked as upper limits because of the uncertainties in the radio slope and hence in the contribution of a jet. XSS J1227 appears to be rather away from the NS binaries locus and, in case of a jet in this system, it is closer to the $\mathrm{BH}$ correlation. Its position could move upwards, matching at $10 \mathrm{kpc}$ the transition region

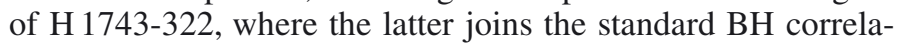
tion. While we cannot infer the true nature of the compact star in XSS J1227 based on this diagram alone, if a jet is present, it could possibly favour a BH LMXB. If so and similarly for H 1743-322 (Coriat et al. 2011), this binary would be in an extremely deep low hard state, where the accretion flow becomes radiatively inefficient. This flow is then dominated by advection in which a significant fraction of the energy is advected and not

3 The radio-vs.-X-ray luminosity diagram is constructed using data for GX 339-4 from Corbel et al. (2003) and a distance of $8 \mathrm{kpc}$, for H 1743322 from Table 1 of Coriat et al. (2011) and distance of $8 \mathrm{kpc}$, and for V404 Cyg from Corbel et al. (2008) and a distance $2.39 \mathrm{kpc}$. For the atoll NS binary Aql X-1, we use data from Miller-Jones et al. (2010) and distance $5 \mathrm{kpc}$, and for 4U 1728-34 we use data from Migliari \& Fender (2006) and distance $4.6 \mathrm{kpc}$. 
radiated away (Narayan \& Yi 1994). The energy either crosses the BH event horizon or is expelled in outflows. Systems dominated by jet emission, where most of the energy is channeled in a jet, emit X-rays at the base of the jet. These are produced either by synchrotron emission or by inverse Compton scattering by outflowing particles. In the previous section, we derived an approximate estimate of the size of the X-ray emitting region, which is much smaller than that of typical ADC in LMXBs. Coriat et al. (2011) also suggest that $\mathrm{H}$ 1743-322 possesses two components, one radiatively efficient and one inefficient, which co-exist and dominate alternatively in the X-ray band during the hard state. In the case of XSS J1227, the bulk of X-rays does not seem to originate in a jet because of the evidence of accretion. Further radio data would be important to assess the true nature of the radio emission.

\section{Conclusions}

We have presented new X-ray data to study the long-term history of XSS J1227 and to infer whether the X-ray emission is variable on timescales down to msec in order to shed light on the nature of this peculiar source. We here summarize the main conclusions:

- The long-term X-ray history of XSS J1227 over a timespan of $7 \mathrm{yr}$ shows that it is a persistent low X-ray luminosity source and not a transient one. The associated source 2FGL J1227 is also found to be a steady high-energy gamma-ray source over an overlapping period of $4.7 \mathrm{yr}$. The co-existence of X-ray and gamma-ray emissions does not favour an MSP binary nature if the two sources are associated.

- XSS J1227 displays flares and dips in all of the X-ray observations, with flares possibly occurring on a timescale of $9-11 \mathrm{~h}$ and duration of $\sim 1 \mathrm{~h}$. Flares are grouped in multiple events. We confirm previous finding of the occurrence of flare-dip pairs. The spectral hardening in these dips are due to an intervening dense, $N_{\mathrm{H}} \sim 4 \times 10^{21} \mathrm{~cm}^{-2}$, absorber covering $\sim 60 \%$ of the $\mathrm{X}$-ray source. Intense dipping activity is found during quiescence. These dips have variable lengths from $\sim 10 \mathrm{~s}$ to $1100 \mathrm{~s}$. A marginal spectral softening is detected in the longer dips. We estimate the size of the X-ray emitting region to be $\sim 2-8 \times 10^{8} \mathrm{~cm}$, much smaller than those derived for LMXB ADC dippers. The UV flares and dips are found to occur simultaneously with the X-ray ones.

- We searched for fast pulsations down to msec in the fasttiming X-ray data obtaining upper limits to fractional pulse amplitudes of 15-25\%. These do not exclude an MSP in this system. A rotation-powered MSP could be favoured by the SED at gamma-ray energies and possibly at radio frequencies, but not from the X-ray and UV/optical/nIR data. The $\mathrm{X}$-ray source is consistent with being an accretion-powered binary.

- From the combined UV/optical/nIR spectrum, we find evidence of a hot component at $\sim 13 \mathrm{kK}$ and a cool one at $\sim 4.6 \mathrm{kK}$. The former is consistent with an X-ray-heated accretion region, which is smaller than that of a tidally truncated accretion disc but larger than those found in other LMXBs with either NS or BH. The cool component would suggest a late-type K2-K5 companion star and in turn a distance of $2.4-3.6 \mathrm{kpc}$. Then the orbital period would be in the range of $7-9 \mathrm{~h}$. This would embrace the $4.3 \mathrm{~h}$ periodicity previously inferred (dM10) if it were the first harmonic. A $20 \%$ variability at $6.4 \mathrm{~h}$ is found in the quiescent near-UV photometry, further suggesting a long orbital period.
- The X-ray-to-UV luminosity ratio is compatible with an LMXB hosting an NS that is much larger than that observed in BH LMXBs. On the other hand, the uncertainties in the radio spectral slope may also allow the possibility of a compact jet. In this case, the radio and X-ray luminosities might indicate XSS J1227 as a BH binary rather than an NS atoll system during island states. The persistent low luminosity of this source could be due to a radiatively inefficient accretion flow, where the radio emission originates in a jet but the X-ray emission does not. If a BH binary, XSS J1227 were the first LMXB to be associated with a high-energy gamma-ray source.

To further progress on the intriguing nature of this source, it is crucial both to obtain new observations in order to infer whether flares also occur in the radio domain and to study the radio spectrum and optical spectroscopy in order to determine the true orbital period.

Acknowledgements. D.d.M. and T.B. acknowledge financial support from ASI under contract ASI/INAF I/009/10/0. A.P. acknowledges the support of the grants AYA2012-39303, SGR2009- 811, and iLINK2011-0303. This research has received funding from the European Commnunity's Seven Framework Programme (FP7/2007-2013) under grant agreement ITN 215212. This work made use of data supplied by the UK Swift Science Data Centre at the University of Leicester. D.d.M. wishes to thank Dr. Norbert Schartel and the ESAC staff for their help in obtaining the XMM-Newton data.

\section{References}

Abdo, A., Ackermann M., Ajello, M. et al. (Fermi LAT Collaboration) 2009a, ApJ, 701, 123

Abdo, A., Ackermann M., Ajello, M. et al. (Fermi LAT Collaboration) 2009b, ApJ, 706, 56

Abdo, A., Ackermann M., Ajello, M. et al. (Fermi LAT Collaboration) 2009c, Science, 325,840

Ackermann, M., Ajello, M., Ballet, J. et al. (Fermi LAT Collaboration) 2012, Science, 335, 189

Archibald, A., Kaspi, V., Bogdanov, S., et al. 2010, ApJ, 722, 88

Bogdanov, S., Grindlay, J., Heinke, C., et al. 2006, ApJ, 646, 1104

Bogdanov, S., Archibald, A., Hessels, J., et al. 2011, ApJ, 742, 97

Boirin, L., Diaz Trigo, M., Parmar, A., \& Kaastra, J. 2005, A\&A, 436, 195

Bradt, H., Rothschild, R., \& Swank, J. 1993, A\&AS, 97, 355

Butters, O. W., Norton, A. J., Hakala, P. J., Mukai, K., \& Barlow, E. 2008, A\&A, 487, 271

Church, M., \& Balucinska-Church, M. 2004, MNRAS, 348, 995

Corbel, S., Nowak, M., Fender, R., Tzioumis, A., \& Markoff, S. 2003, A\&A, 400, 1007

Corbel, S., Koerding, E., \& Kaaret, P. 2008, MNRAS, 389, 1697

Coriat, M., Corbel, S., Prat, L., et al. 2011, MNRAS, 414, 677

de Martino, D., Falanga, M., Bonnet-Bidaud, J., et al. 2010, A\&A, 515, A25

Diaz Trigo, M., Parmar, A., Boirin, L., et al. 2009, A\&A, 493, 145

Evans, P. A., Beardmore, A., Osborne, J., et al. 2009, MNRAS, 397, 1177

Feroci, M., Costa, E., Soffitta, P., et al. 2007, Nucl. Instr. and Meth., 581, 728

Froning, C., Cantrell, A., Maccarone, T., et al. 2011, ApJ, 743, 26

Gallo, E. 2010, in LNP 794, The The Jet Paradigm: From Microquasars to Quasars, ed. T. Belloni, 85

Gallo, E., Fender, R., \& Pooley, G. 2003, MNRAS, 344, 60

Hadasch, D., Torres, D., Tanaka, T., et al. 2012, ApJ, 749, 54

Hill, A., Szostek, A., Corbel, S., et al. 2011, MNRAS, 415, 235

Hynes, R., \& Robinson, E. 2012, ApJ, 749, 3

Iaria, R., Lavagetto, G., D'Ai', A., Di Salvo, T., \& Robba, N. 2007, A\&A, 463, 289

Israel, G. L., \& Stella, L. 1996, ApJ, 468, 369

Johnston, H. M., \& Kulkarni, S. R. 1991, ApJ, 368, 504

Kaplan, D., Stovall, K., Ransom, S., et al. 2012, ApJ, 753, 174

Kerr, M., Camilo, F., Johnson, T., et al. 2012, ApJ, 748, 3

Knigge, C. 2006, MNRAS, 373, 484

Kong, A., Huang, R., Takata, J., et al. 2012, ApJ, 747, 3

Masetti, N., Morelli, L., Palazzi, E., et al. 2006, A\&A, 459, 21 
A\&A 550, A89 (2013)

Mason, K. O., Breeveld, A., Much, R., et al. 2001, A\&A, 365, 36 Migliari, R., \& Fender, R. 2006, MNRAS, 366, 79

Migliari, R., Fender, R., Rupen, M., et al. 2003, MNRAS, 342, 67

Miller-Jones, J., Sivakoff, G., Altamirano, D., et al. 2010, ApJ, 716, 109

Narayan, R., \& Yi, L. 1994, ApJ, 428, 13

Nolan, P., Abdo, A., Ackermann, M., et al. 2012, ApJS, 199, 31

Papitto, A., Torres, D., \& Rea, N. 2012, ApJ, 756, 188

Patruno, A., \& Watts, A. 2012, in Timing neutron stars: pulsations, oscillations and explosions, eds. T. Belloni, M. Mendez, \& C. Zhang, ASSL, in press [arXiv: 1206.2727]

Pretorius, M. 2009, MNRAS, 395, 386

Ransom, S. M., Ray, P., Camilo, F., et al. 2011, ApJ, 727, 16

Romani, R., \& Shaw, M. 2011, ApJ, 743, 26

Saitou, K., Tsujimoto, M., Ebisawa, K., \& Ishida, M. 2009, PASJ, 61, 13
Saitou, K., Tsujimoto, M., Ebisawa, K., et al. 2011, PASJ, 63, 759

Smith, D., \& Dhillon, V. 1998, MNRAS, 301, 767

Strüder, L., Briel, U., Dennerl, K., et al. 2001, A\&A, 365, 18

Takata, J., Cheng, K., \& Taam, R. 2012, ApJ, 745, 100

Tam, P., Hui, C., Huang, R., et al. 2010, ApJ, 724, 207

Tavani, M., Argan, A., Boffelli, A., et al. 2009a, A\&A, 502, 995

Tavani, M., Bulgarelli, A., Piano, G., et al. 2009b, Nature, 462, 620

Torres, D., Rea, N., Esposito, P., et al. 2012, ApJ, 744, 106

Turner, M. J. L., Abbey, A., Arnaud, M., et al. 2001, A\&A, 365, 27

van der Klis, M. 1989, in Timing Neutron Stars, eds. H. Ögelman, \& E. P. J. van den Heuvel, 27

Vaughan, B. A., van der Klis, M., Wood, K. S., et al. 1994, ApJ, 435, 362

Wood, K. S., Norris, J. P., Hertz, P., et al. 1991, ApJ, 379, 295

Wu, J., Kong, A., Huang, R., et al. 2012, ApJ, 748, 141 